\title{
Article
}

\section{Temporal evolution of solar energetic particle spectra}

Doran, Donald Joseph and Dalla, Silvia

Available at http://clok.uclan.ac.uk/14984/

Doran, Donald Joseph and Dalla, Silvia ORCID: 0000-0002-7837-5780 (2016) Temporal evolution of solar energetic particle spectra. Solar Physics, 291 (7). pp. 2071-2097. ISSN 0038-0938

It is advisable to refer to the publisher's version if you intend to cite from the work. http://dx.doi.org/10.1007/s11207-016-0956-4

For more information about UCLan's research in this area go to

http://www.uclan.ac.uk/researchgroups/ and search for <name of research Group>.

For information about Research generally at UCLan please go to http://www.uclan.ac.uk/research/

All outputs in CLoK are protected by Intellectual Property Rights law, including Copyright law. Copyright, IPR and Moral Rights for the works on this site are retained by the individual authors and/or other copyright owners. Terms and conditions for use of this material are defined in the policies page.

\section{CLoK}

Central Lancashire online Knowledge www.clok.uclan.ac.uk

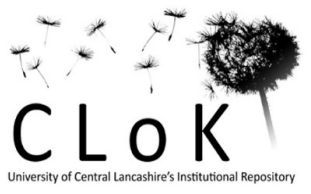




\title{
Temporal Evolution of Solar Energetic Particle Spectra
}

\author{
Donald J. Doran ${ }^{1}$. Silvia Dalla ${ }^{1}$
}

(c) Springer

\begin{abstract}
During solar flares and coronal mass ejections, solar energetic particles $(\mathrm{SEP})$ may be released into the interplanetary medium and near-Earth locations. The energy spectra of SEP events at 1 AU are typically averaged over the entire event or studied in a few snapshots. In this paper we analyze the time evolution of the energy spectra of four large selected SEP events using a large number of snapshots. We use a multi-spacecraft and multi-instrument approach for the observations, obtained over a wide SEP energy range. We find large differences in the spectra at the beginning of the events as measured by different instruments. We show that over time, a wave-like structure is observed traveling through the spectra from the highest energies to the lowest energies, creating an "arch" shape which then straightens into a power law later in the event, after times of the order of 10 hours. We discuss the processes that determine SEP intensities and their role in shaping the spectral time evolution.
\end{abstract}

Keywords: perpendicular transport, drift, cross-field transport,

\section{Introduction}

Solar energetic particles (SEPs) are ions and electrons that have been accelerated from the coronal and solar wind plasma by solar flares and coronal mass ejections (CMEs). SEP ions consist mainly of protons, with further small numbers of heavier ions from helium up to iron, and even heavier species. SEPs are of particular importance for scientific and space exploration missions, because they are a radiation hazard to spacecraft and to human occupants. Of these radiation hazard particles, protons are the most abundant, and therefore present the most serious safety risk. In this regard, human occupants can suffer cumulative

\footnotetext{
$\triangle$ Dalla

sdalla@uclan.ac.uk

1 University Central Lancashire, Preston, United Kingdom

1 University Central Lancashire, Preston, United Kingdom
} 
radiation dosages causing serious health issues, and spacecraft can experience irreversible damage to their functional electronics, (Crosby, Heynderickx, and Jiggens, 2013).

SEP events are typically studied by analyzing the profiles of particle intensity versus time. Multi-spacecraft observations have elucidated how SEP profiles and their properties depend on the location of the observing spacecraft with respect to the associated solar events (Cane, Reames, and von Rosenvinge, 1988; Kallenrode et al., 1993; Reames, Barbier, and Ng, 1996; Richardson et al., 2014). It is well known that near-Earth spacecraft are magnetically connected to solar locations on the western limb, therefore events within W20-W80 have the largest probability to produce SEP events at Earth. The role of latitudinal separation between observers and solar source regions in determining whether or not an SEP event will be observed is less clear (Dalla and Agueda, 2010).

The long standing interpretation of the transport of SEPs from the corona through the heliosphere has assumed propagation to be along the interplanetary magnetic field (IMF) lines, without transport of particles perpendicular to the field. When particles are accelerated by CME-driven shocks, the region of injection is very extended, providing a possible explanation for the fact that SEPs are often observed by spacecraft at large longitudinal separation from the source active region at the Sun. However recent observations of impulsive SEP events by widely separated spacecraft (Wiedenbeck et al., 2013), seem to imply significant cross-field transport, or movement across the interplanetary magnetic field lines, since for this type of event, the injection is thought to take place in a relatively compact flare region.Dresing et al. (2014) analyzed a number of SEP events observed by STEREO and concluded that one possible mechanism for the wide-spread type SEP event is efficient perpendicular transport in the interplanetary medium (IM), while another scenario is a wide particle spreading occurring close to the Sun, and probably both mechanisms are at work for many SEP events. Among possible mechanisms for transport across the field, recent work has shown thatdrifts due to gradient and curvature of the interplanetary magnetic field are significant (Marsh et al., 2013)). Drift takes place in both scatter-free and scattering modes, and is a function of particle energy as well as the location of the particle source at the Sun (Dalla et al., 2013; Kelly, Dalla, and Laitinen, 2012). Drift of SEPs also produces deceleration because particles move anti-parallel to the solar wind electric field, a process that has been termed drift-induced deceleration (Kóta, 1979; Dalla, Marsh, and Laitinen, 2015).

Energy spectra, showing the particle intensity at a given time versus the average energy of each instrument channel, provide an alternative view of SEP events. Although spectra have been analyzed for a variety of SEP events, in most studies a single representative spectrum is shown for an entire event. Event integrated spectra tend to exhibit a power law-dependence, which for large shockassociated gradual events almost always yields a double power law, sometimes called a knee. According to Li et al. (2009), the energy break or rollover feature depends on $(Q / A)^{\alpha}$, where $Q$ is the ionic charge and $A$ is the ion mass in atomic mass units, with the power law index $\alpha$ ranging between $\approx 1$ and $\approx 2$. The $Q / A$ dependence of the break energies however, is not always easy to determine due to the fact that the charge state is very often uncertain, as shown by Mewaldt 
et al. (2012) who presented event integrated proton spectra for 16 GLE events over an energy range from $0.3 \mathrm{MeV}$ to $\approx 700 \mathrm{MeV}$.

Reames, Kahler, and Ng (1997) considered energy spectra at three times during an SEP event observed by multiple spacecraft, and showed that so-called invariant spectra are observed in the decay phase of the event, where the different spectra of each observing spacecraft become very similar to each other. A new approach to the time evolution of the energy spectra of SEPs was proposed by Laurenza et al. (2012). The authors used the Shannon differential entropy to study the time evolution of a particle event measured by the SOHO ERNE instrument in the proton energy range of $1.67-112 \mathrm{MeV}$.

In this paper we analyze the time evolution of SEP spectra using a large number of snapshots and instrumentation from multiple spacecraft. We consider data for some historical events which took place in the 1970s as well as more recent events observed by STEREO. Our focus is on large events with strong flux at both the low, and high energy ends of the SEP range, to ensure that many spectral points with good statistics are available. We discuss the observations within the framework of the current understanding of SEP acceleration and propagation. We also discuss spectral indices for these events, comparing the event averaged-spectra with those late in the event after the spectral break when the "arch" structure is no longer present in the spectra.

\section{Observations}

The types of data to be used for this project include various previously compiled lists of SEP events and actual data collected by a variety of spacecraft. The type of events of particular interest are those with high flux at the low energy channels e.g. 5-15 MeV, and having at the same time high flux at the high energy channels e.g. $>100 \mathrm{MeV}$. In this paper we focus on SEP protons using nine spacecraft and their instruments, as highlighted below and also detailed in Table 1:

- ACE, (Advanced Composition Explorer) with instruments ULEIS (Ultra Low Energy Isotope Spectrometer, 0.6 to $8.7 \mathrm{MeV} / n$ energy range) and SEPICA (Solar Energetic Particle Ionic Charge Analyzer, 0.4 to $6.0 \mathrm{MeV} / n$ energy range) at web site http://www.srl.caltech.edu/ACE/. See Stone et al. (1998).

- GOES 11 and 13 (Geostationary Operational Environment Satellites), with instruments SEM/ESP (Space Environment Monitor and Energetic Particle Sensor) at web site www.swpc.noaa.gov/Data/goes.html. See Onsager et al. (1996); McCuistion and Bengston (1996).

- HELIOS 1 and 2, E6 experiment, Energetic Electron and Proton Detector (low energy telescope at 4-51 MeV/n energy range), and E7 experiment (high energy telescope at 3.4-206.5 MeV energy range), at web site http://nssdc.gsfc.nasa.gov/nmc/spacecraftDisplay. See Porsche (1981).

- IMP-8 GME (Goddard Medium Energy Experiment) also called Explorer 50 and IMP-J at web site http://spdf.gsfc.nasa.gov/imp8/GME, and CRNC 
(Cosmic Ray Nuclear Composition Experiment).

at web site http://explorers.gsfc.nasa.gov/missions.html.

- SOHO (The Solar and Heliospheric Observatory) ERNE (Energetic and Relativistic Nuclei and Electron experiment) at web sites http://soho.esac.esa.int/data/data.html, or http://soho.nascom.nasa.gov/about/about.html. See Torsti et al. (1995).

- STEREO A (ahead of the Earth) and STEREO B (behind the Earth), (Solar TErrestrial RElations Observatory) IMPACT instrument (In-situ Measurement of Particles and CME Transients), LET (Low Energy Telescope), See Mewaldt et al. (2008), and HET (High Energy Telescope), See von Rosenvinge et al. (2008) at web site http://www.nasa.gov/mission-pages/stereo/main/index.html.

- WIND (Comprehensive Solar Wind Laboratory for Long Term Solar Wind Measurements) 3DP SST instrument (3 Dimensional Plasma Analyzer Solid State Telescope) measuring protons up to $6 \mathrm{MeV} / \mathrm{n}$, at web site http://http://www.wind.nasa.gov/, See Lin et al. (1995).

Table 1. Spacecraft and instruments used for data acquisition

\begin{tabular}{lllllr}
\hline Spacecraft & Instrument & $\begin{array}{l}\text { Proton } \\
\text { Energy, MeV }\end{array}$ & $\begin{array}{l}\text { Start } \\
\text { Date }\end{array}$ & $\begin{array}{l}\text { End } \\
\text { Date }\end{array}$ & Orbit \\
\hline ACE & SEPICA & $0.4-6.0$ & Aug 1997 & active & L1 \\
ACE & ULEIS & $0.6-8.7$ & Aug 1997 & active & L1 \\
GOES 11 & ESP & $0.6-500$ & May 2000 & Dec 2011 & Geostationary \\
GOES 13 & ESP & $0.7-500$ & Jun 2006 & active & Geostationary \\
HELIOS 1 & E6 & $4.0-51.0$ & Jan 1975 & Feb 1985 & Heliocentric \\
HELIOS 1 & E7 & $3.4-206.5$ & Jan 1975 & Feb 1985 & Heliocentric \\
HELIOS 2 & E6 & $4.0-51.0$ & Jul 1976 & Dec 1979 & Heliocentric \\
HELIOS 2 & E7 & $3.4-206.5$ & Jul 1976 & Dec 1979 & Heliocentric \\
IMP 8 & CRNC & $10.9-95.0$ & Oct 1973 & active & Geostationary \\
IMP 8 & GME & $0.9-237$ & Oct 1973 & active & Geostationary \\
SOHO & ERNE & $1.8-50.7$ & Dec 1995 & active & L1 \\
STEREO A & LET & $1.8-15.0$ & Oct 2006 & active & Heliocentric \\
STEREO A & HET & $13.6-100$ & Oct 2006 & active & Heliocentric \\
STEREO B & LET & $1.8-15.0$ & Oct 2006 & active & Heliocentric \\
STEREO B & HET & $13.6-100$ & Oct 2006 & active & Heliocentric \\
WIND & 3DP-SST & $0.1-6$ & Nov 1994 & active & L1 now L2 \\
\hline
\end{tabular}

The observational data has been obtained over diverse locations in space, as shown in Table 1, from the near-Earth geocentric views of IMP 8, to HELIOS 1 and 2 orbiting the Sun, to SOHO and ACE just outside the Earth's magnetosphere, and with those of Goes 11 and 13, as well as WIND inside the Earth's magnetosphere, and finally to the stereoscopic views of STEREO A and B. Several different space environments are observed by the IMP 8 instruments as the spacecraft passes through the bow shock, magnetosphere, magnetotail, 
and the solar wind in its geocentric orbit of 25 to 45 Earth radii. In each orbit of 12.5 days, it spends only 7-8 days in the solar wind, but nevertheless provides much-needed information on particle flux at the near-Earth position.

Four widely different SEP events have been examined, starting with their individual time-intensity plots, and followed by a study of the time evolution of their spectra. The first of these events is associated with a single flare (No. 1 of Table 2), but there may have been a CME also since there were no CME observations in 1977. The second event is due to both a CME and an associated flare (No. 2a and No. 2b) of Table 2). The third event is a complicated series of four CMEs and associated flares (No. 3a and No. 3b), but we focus only on the third peak of Figure 9. Finally, the fourth and last event is a series of two CMEs and associated flares (No. 4a and No. 4b), but we focus only on peak A of Figure 14.

Table 2. Properties of Solar Events Associated With The Four Studied SEP Events, showing the start times of the flare and CME, not the start time of the event, and with NAR being the NOAA active region number.

\begin{tabular}{ccccccr}
\hline No. & $\begin{array}{c}\text { Event } \\
\text { Date }\end{array}$ & $\begin{array}{c}\text { Flare/CME } \\
\text { start time }\end{array}$ & Type & Location & NAR & $\begin{array}{r}\text { X-ray } \\
\text { class }\end{array}$ \\
\hline 1 & $1977-11-22$ & $21: 30$ & flare & N23W40 & X1 \\
2a & $2000-07-14$ & $10: 03$ & flare & N22W07 & 9077 & X5.7 \\
2b & $2000-07-14$ & $10: 54$ & CME & N20W08 & & halo \\
3a & $2001-04-15$ & $13: 19$ & flare & S20W85 & 9415 & X14.7 \\
3b & $2001-04-15$ & $14: 06$ & CME & S20W90 & & halo \\
$4 \mathrm{a}$ & $2012-03-07$ & $02: 00$ & flare & N17E15 & 11429 & X5.7 \\
$4 a$ & $2012-03-07$ & $00: 24$ & CME & N22E12 & & halo \\
\hline
\end{tabular}

\section{Selection of SEP Rvents For Study}

We selected four SEP events among those with an intensity $>10^{2}$ as measured in particles $\mathrm{cm}^{-2} \mathrm{~s}^{-1} \mathrm{sr}^{-1}(\mathrm{MeV} / \mathrm{nuc})^{-1}$ in the $10-15 \mathrm{MeV}$ energy channel as well as 0.1 intensity in the 138-200 MeV channel, over the time range of 1976-2012. A further criterion was added that the time-intensity profile of the event should resemble the so-called classic profile whereby the particle flux rises sharply followed by a steady and even decay over a period of 2 to 4 days. It was necessary to apply this requirement as some of the events selected for study, although very intensive and energetic, were nevertheless very complicated in their profiles with several spikes and irregularities in their decays. A final list of 14 events satisfied the above selection criteria during 1976 to 2012, and of these, four were chosen for detailed study.

The time-intensity profiles for a near-Earth observer for the selected events were obtained from ESA's Solar Energetic Particle Environment Modeling (SEPEM) application server, see (Crosby et al., 2015). This server uses data 
compiled from the IMP-8/GME and the GOES 7-13/SEM/EPS spacecraft covering the period November 1973 to March 2012 to generate the so-called SEPEM reference proton data set. The advantage of using the dataset is that the SEP fluxes have been inter-calibrated and de-spiked, producing a uniform dataset. In addition, the SEPServer data server, see (Vainio et al., 2013) was used for the HELIOS 1 and 2, STEREO A/B, and SOHO/ERNE plots.

Spectra were plotted with a resolution of 1 to 2 hours over the course of the event. Spectral plots were made using The Virtual Energetic Particle Observatory (VEPO) analysis tool, which operates within the domain of the Virtual Heliospheric Observatory (VHO). VEPO has two interfaces for generating spectral plots of energetic particle fluxes from multiple instruments flown on multiple spacecraft. The fluxes are averaged over user-specified time spans. We used the Multi-Source Spectral Plots-1, (MSSP-1), which provides spectral plots of H and He for 30 data sources.

\section{Results and Data: Time-intensity and Spectral Plots of Four Selected SEP Events}

\subsection{GLE Event of 1977-11-22}

The event of 1977-11-22 is from the early history of SEP investigations, and although there is no data from the SolarMonitor, CDAW, or HELIO catalogs, however all the literature publications seem to agree on the flare identification of the event. This is clearly the only strong SEP event for the month of November, 1977, making it a good choice for the study of its properties without interference from preceding or following flares or CMEs. The time intensity profile is shown in Figure 1, covering 30 days with $\approx 3.65$ days per vertical line. Figure 2 , is an expanded view covering 3 days and 14 hours, showing a smooth profile and a flux of 230 particles $\mathrm{cm}^{-2} \mathrm{~s}^{-1} \mathrm{sr}^{-1}(\mathrm{MeV} / \mathrm{nuc})^{-1}$ in the $5-7 \mathrm{MeV}$ channel (red) and 0.09 in the $138-200 \mathrm{MeV}$ channel (yellow). This event had a strong flare according to Fenton, Fenton, and Humble (1978); Burlaga et al. (1980) and Chambon et al. (1981), which created relativistic protons that were observed as a ground level event (GLE) on 16 neutron monitors. The flare occurred on the rising portion of the 21st sunspot cycle, and was the first clear event for several years. The location of the source was given as N23W40 by Komori (1979) and Gombosi et al. (1979), who observed the event from the PROGNOZ 6 satellite which was outside the Earth's magnetosphere at the time. The authors reported proton fluxes from $2-500 \mathrm{MeV}$. Several authors reported that the time intensity variations of the SEPs during the event could be described by a simple diffusion model, mainly due to the fact that this event took place during quiet conditions in the IMF and low solar particle background. One reason for the choice of this event, is the fact that it was during the short time period 1976-1979, when both HELIOS 1 and 2 were in operation. At the time HELIOS 1 was at solar ecliptic (SE) longitude $18.3^{\circ}$, at a distance of $0.64 \mathrm{AU}$, and HELIOS 2 was at $48.7^{\circ} \mathrm{SE}$ longitude, at a distance of $0.58 \mathrm{AU}$ from the Sun as calculated by (http://omniweb.gsfc.nasa.gov/coho/helios/heli.html). The relative positions of 
the two spacecraft with the Earth in the solar ecliptic frame is shown in the small diagram at the bottom of the GOES time-intensity plot of Figure 2.

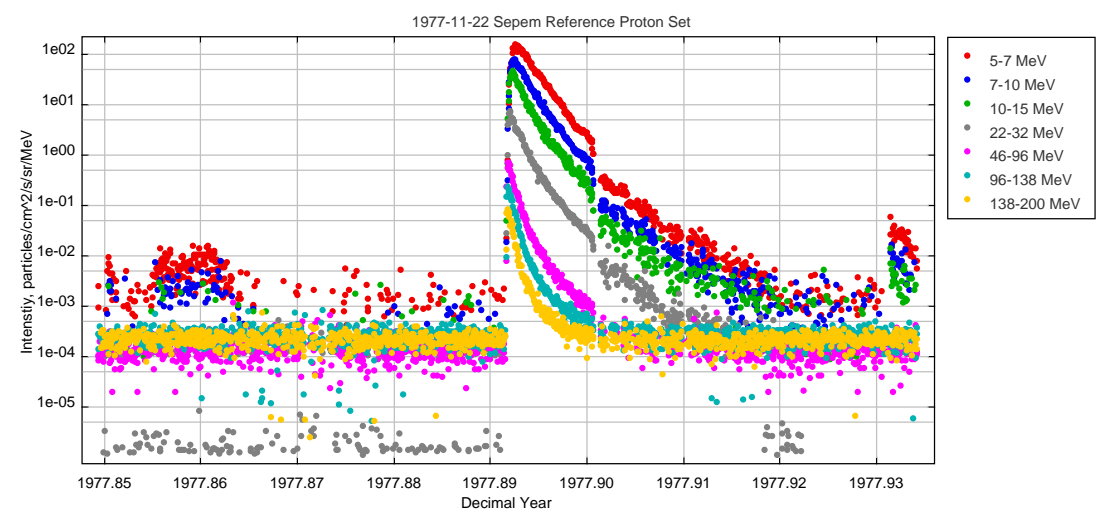

Figure 1. 1977-11-22 SEP Event Time-intensity Profile. In this image, the intensities of protons are shown for energy channels 5-7 MeV (red), 7-10 MeV (blue), 10-15 MeV (green), 22-32 MeV (gray), 66-95 MeV (pink), 96-138 MeV (cyan), and 138-200 MeV (yellow) for one month during November and December, 1977.

The spectral plots for this event are shown in a montage in Figure 3, where each panel corresponds to a 1 hour accumulation time that falls within one of the intervals delimited by vertical lines in Figure 2. In the montage the spectra for HELIOS 1 and 2 (instruments E6 and E7), and IMP-8 (instruments CRNC and GME) are plotted at subsequent times going from left to right, top to bottom. The time for each image is given on the bottom in bold type, for example in the first plot the time frame is 1977112208 to 1977112209 or 1 hour, together with the date/time format of yyyymmddhh, with yyyy being the year, $\mathrm{mm}$ the month, dd the day, and hh the hour on a 24 hour clock. This plot represents the background intensity. The second plot is 1977112209 to 1977112210,1 hour later, and represents the first change in the spectra above the background spectra. This would put this image at segment No. 2 of the time-intensity plot of Figure 2, at the beginning of the flux enhancement or the onset of the SEP event at 11:00. The peak intensity spectrum for the low energies would be in segment No. 3, 1977112217 to 1977112218 , panel 3.4 , actually at $18: 30$. We have plotted each 1 hour change in the spectra in panels $2.5,2.6,2.7,2.8$, and 2.9, until finally jumping ahead to the last panel No. 10.5 where the spectra return more or less to the background spectrum, and are all very similar. This corresponds to segment No. 10 in Figure 2 at approximately 11:30 November 25th.

The spectral plots shown in the Figure 3 montage, reveal some extraordinary shifts in the energy of the particles. The first panel (No. 2.4) shows the background spectra with the HELIOS 1 E6 (black color) and HELIOS 2 E6 (light blue) and HELIOS 2 E7 (light green), IMP 8 CRNC (red color) and GME (blue color) spacecraft observing approximately the same number of particles in the 7-200 $\mathrm{MeV}$ energy range (with the exception of HELIOS 1 E7 data missing in panels 2.4-2.7). However, in the next panel 2.5 one hour later, which 
is the beginning of the onset of the flux enhancement of the event, the HELIOS spacecraft data show an increase in flux from $10^{-4}$ to $10^{0}$ at all energies but mostly in the higher energies from $20-200 \mathrm{MeV} / \mathrm{n}$. The strange "U" shape in the HELIOS 1 E6 spectra could be due to an instrument failure to record particles in the $20-30 \mathrm{MeV}$ range and is not considered relevant. Also in this panel, a surge of higher energy particles is observed from 50 to $200 \mathrm{MeV} / \mathrm{n}$ of approximately a factor of 10 for the IMP 8 CRNC and GME instruments, but no particles are observed at the lower energies $<50 \mathrm{MeV} / \mathrm{n}$. One hour later, in panel 2.6, the higher energy particles continue to increase for HELIOS 1 and 2, and also the lower energy particles are arriving at HELIOS and IMP 8 . There is also a surge of higher energy particles at IMP 8 CRNC and GME with no particles observed at the lowest energies. In successive panels 2.7, 2.8, and 2.9, the IMP 8 higher energy particles continue to arrive, and the spectra begin to merge with those of HELIOS 1 and 2. In the IMP 8 data, one can describe the time evolution of the spectra as characterized by a "wave" traveling along the spectra from the high energies to the low energies until all energy ranges are populated and the spectra take on a power law shape. Spectra at the earlier times do not display a power law, but have an "arch" shape. The time taken for the wave to travel through the spectra is $\approx 21$ hours (to panel 4.9 ). The lowest energy particles do finally begin to arrive in panel No. 3.4 at IMP $8 \approx 9$ hours after the event onset, and continue to arrive in panel No. 4.9. Panel 4.9 corresponds to just after the time of the peak flux enhancement. The HELIOS spacecraft seem to be better connected to the source of the event and sees higher fluxes earlier at the low energies. 


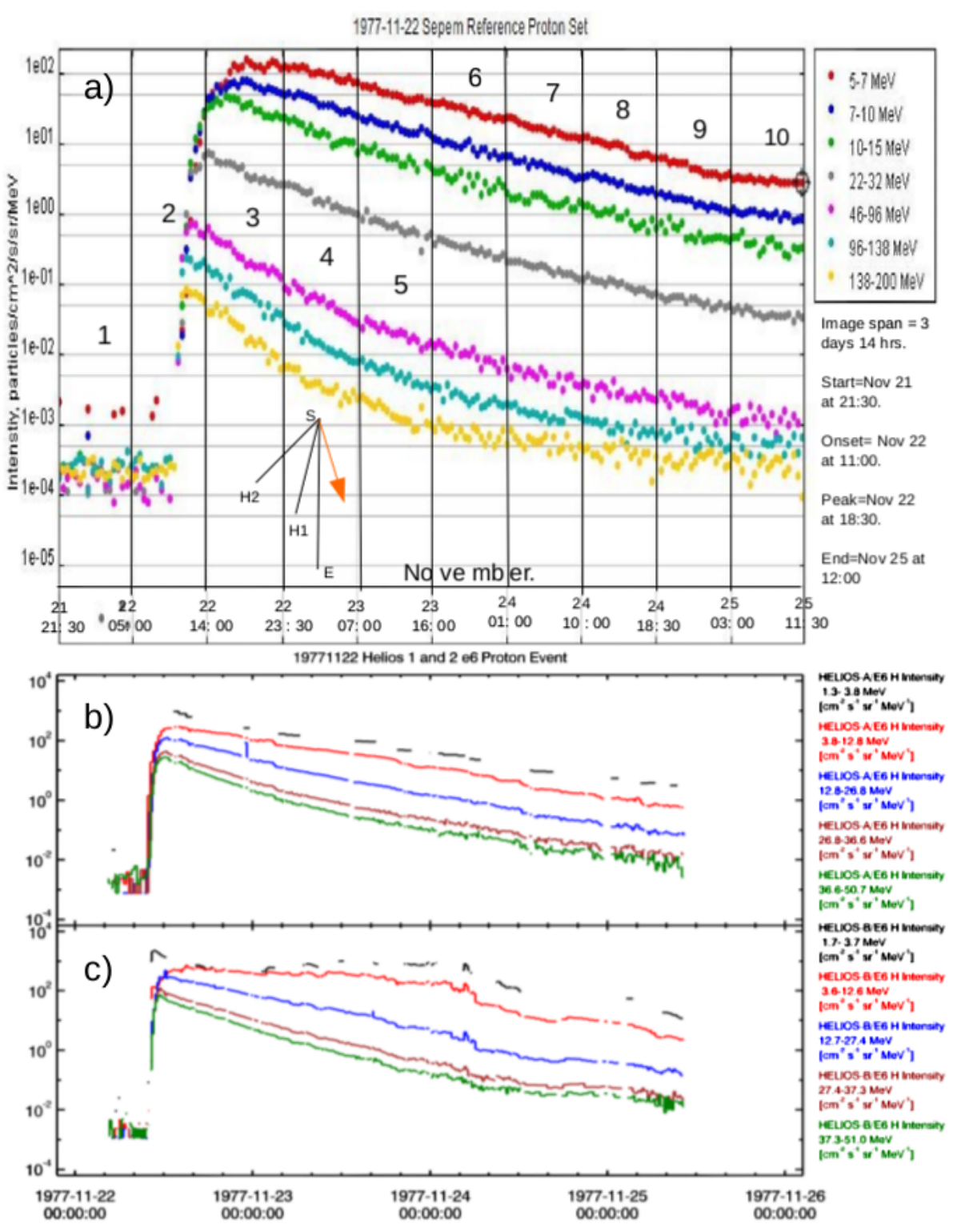

"SEPServer

Figure 2. Time-intensity Profiles of SEP Protons During The 1977-11-22 Event as measured by a) GOES (Crosby, Heynderickx, and Jiggens, 2013), and b) HELIOS 1 and c) HELIOS 2. In this figure, the colors for the GOES plots in the top panel a), are 5-7 MeV (red), $7-10 \mathrm{MeV}$ (blue), $10-15 \mathrm{MeV}$ (green), 22-32 MeV (gray), 46-96 MeV (violet), 96-138 MeV (cyan), 138-200 $\mathrm{MeV}$ (yellow), and the colors for the HELIOS 1 plots in panel b) are, 1.3-3.8 MeV (black), 3.8-12.8 (red), 12.8-26.8 (blue), 26.8-36-6 MeV (cyan), 36.6-50.7 MeV (green), and the colors for the HELIOS 2 plots in panel c) are, 1.7-3.7 (black), 3.6-12.6 (red), 12.7-27.4 MeV (blue), 27.4-37.3 MeV (cyan), 37.4-50.0 MeV (green). The inset in panel a) gives relative positions of the three spacecraft, and the orange arrow indicates the longitude of the flare associated with the event. 
D. J. Doran, S. Dalla
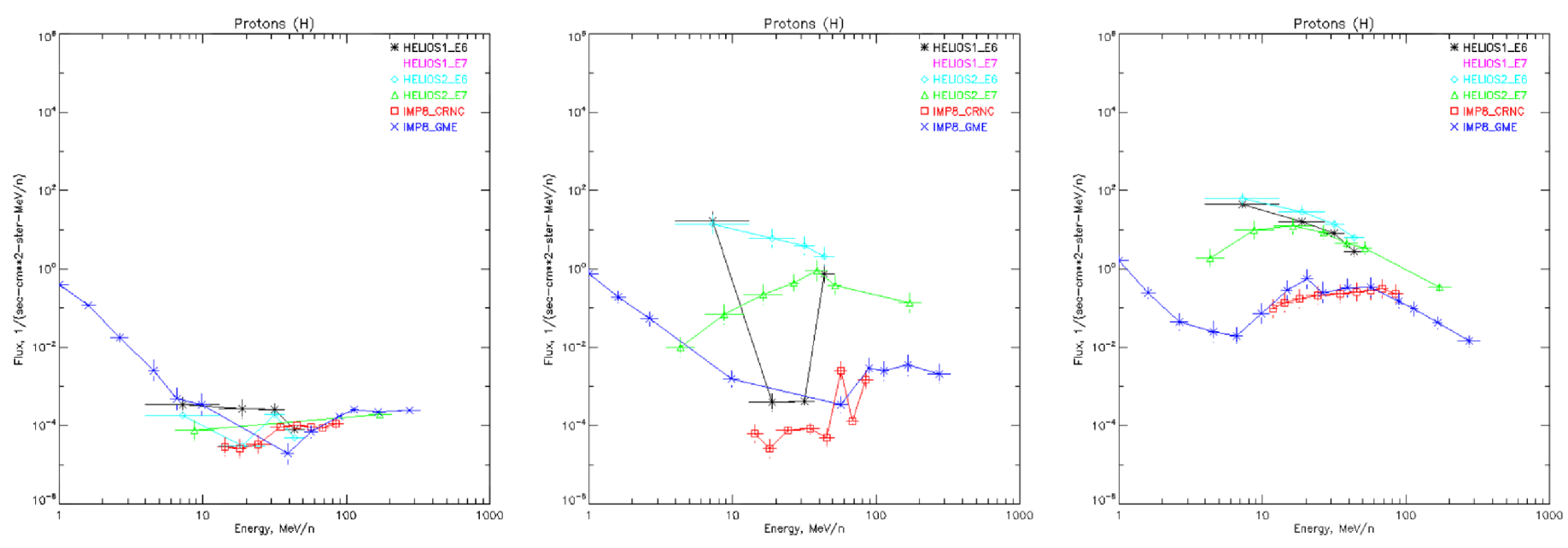

2.4-1977112208_to_1977112209.gif

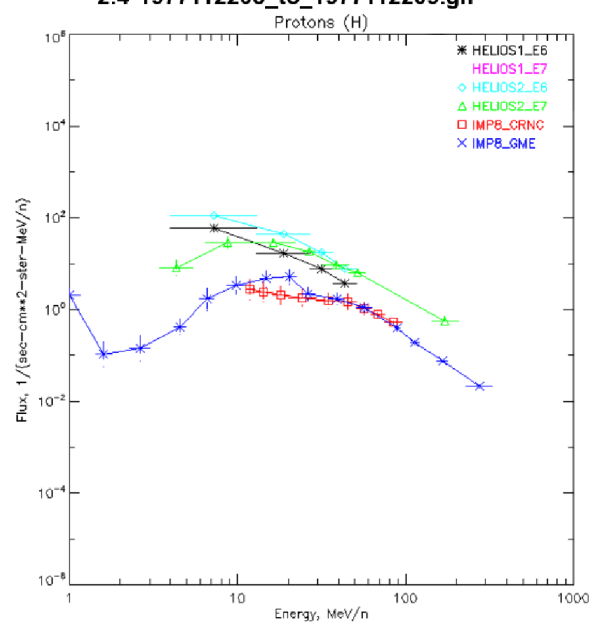

2.5-1977112209_to_1977112210.gif
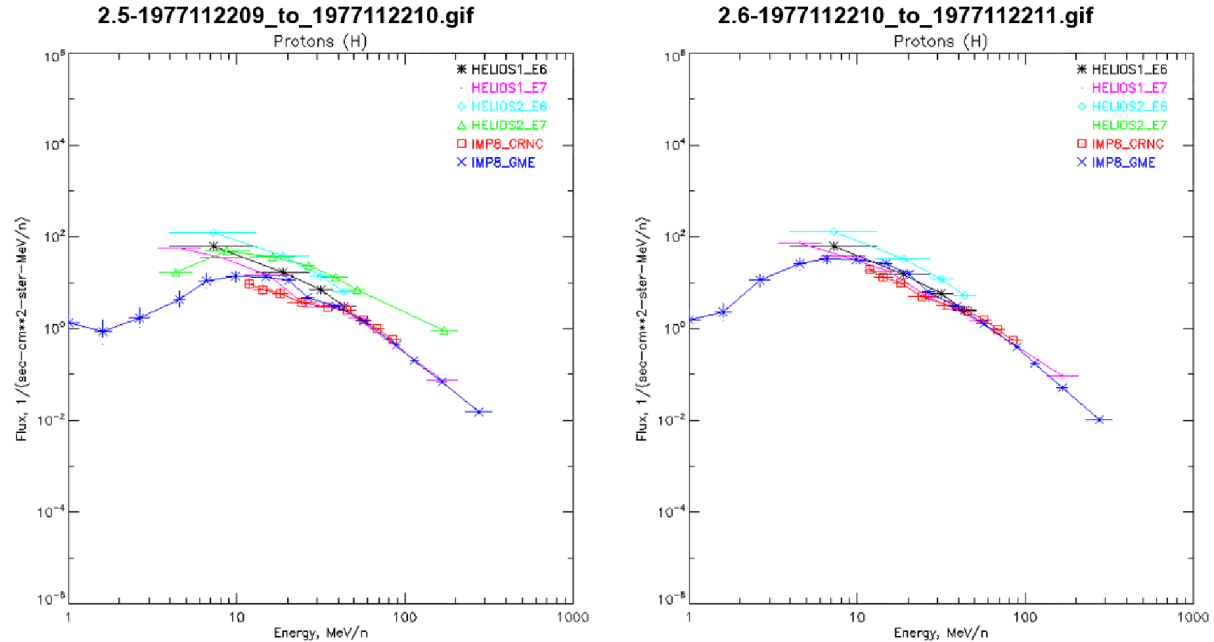

2.7-1977112211_to_1977112212.gif

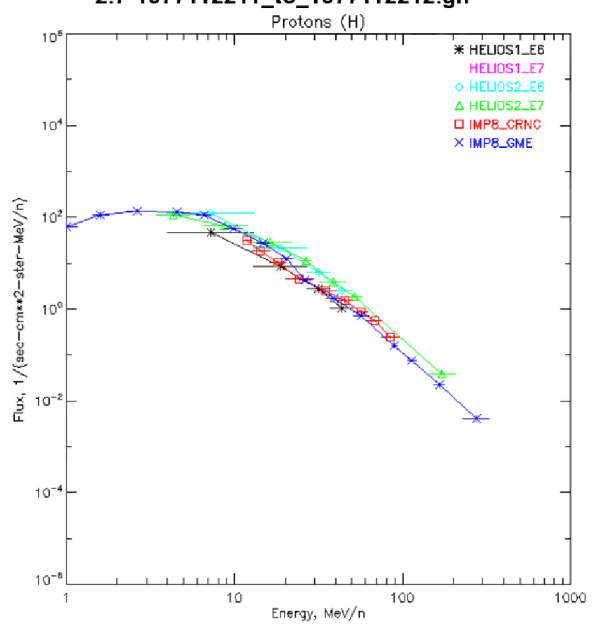

2.8-1977112212_to_1977112213.gif

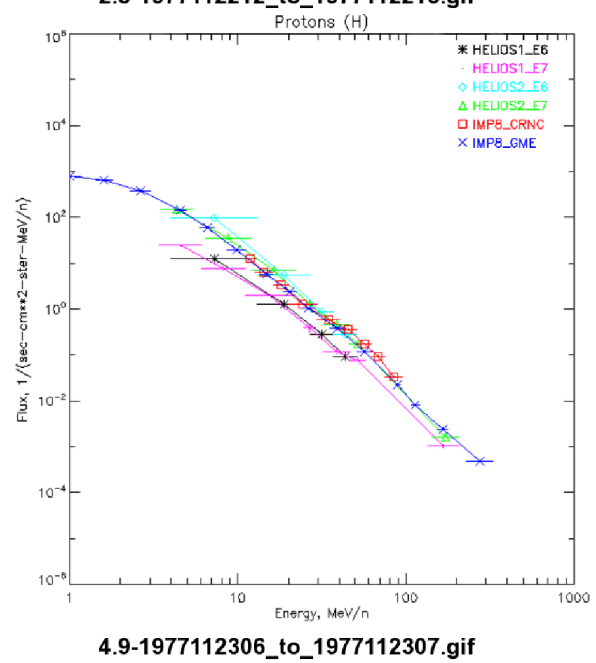

2.9-1977112213_to_1977112214.gif

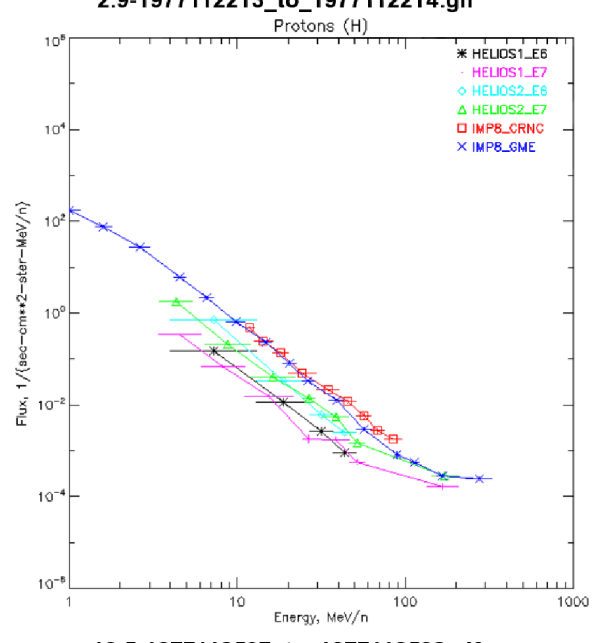

10.5-1977112507_to_1977112508.gif

Figure 3. The Time-evolution of The Energy Spectra of The 1977-11-22 SEP Event measured at multiple spacecraft. The decimal number labeling each plot corresponds to the time interval in Figure 2 a). HELIOS 1 data from E6 and E7 are in black and pink, HELIOS 2 E6 and E7 data are in light blue and green respectively, while IMP 8 CRNC is in red and IMP8 GME is in blue. 
For comparison with the time evolution of spectral snapshots shown in Figure 3, the event averaged spectrum for the three spacecraft of HELIOS 1 and 2 , and IMP 8 over the whole event is shown in Figure 4, where the spectra at different locations are all very similar in a power law shape over the energy range from 1 to $200 \mathrm{MeV}$. This shape agrees well with the spectral shape in the time evolution of the spectra in Figure 3 of panels late in the event e.g. panels 4.9 and 10.5, except that the event averaged spectrum is harder.

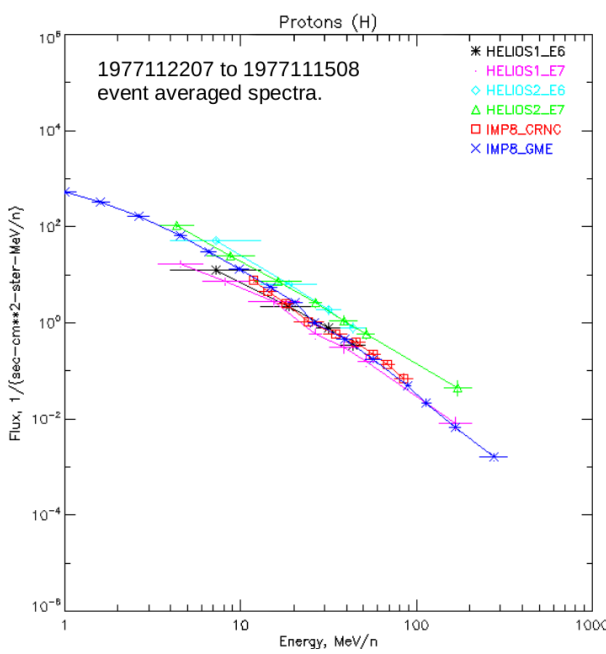

Figure 4. 1977-11-22 to 1977-11-25 Event Averaged Spectra as measured by HELIOS 1 E6 (black color), HELIOS 1 E7 (pink), HELIOS 2 E6 (light blue), HELIOS 2 E7 (green), IMP 8 CRNC (red), and IMP 8 GME (blue)

\subsection{Bastille Day Event of 2000-07-14}

The time-intensity profile for this event is shown in Figure 5, which is the full aspect of the month of July 2000, with only one small event before and two small events after the the main particle flux increase. As reported by the $\mathrm{LASCO}^{1}$ and $\mathrm{HELIO}^{2}$ catalogs, the main event was the combination of a flare followed closely by a CME. The X5.7 flare started at 10:03 on the 14th of July (Bastille Day) at N22W07, while the CME started at 10:54 as a halo event with a velocity of 1674 $\mathrm{km} / \mathrm{sec}$ at N20W08. Together, these events were widely observed at ground level on multiple Earth based neutron monitors, and with particle and magnetic field measurements being made directly with the WIND, ACE, and SOHO spacecraft, as reported by Belov et al. (2001), Klein et al. (2001), and Bieber et al. (2002). Important observations were also reported from the ULYSSES data by Zhang et al. (2003), at which time the spacecraft was at a distance of 3.17 AU from the

\footnotetext{
${ }^{1}$ See http://cdaw.gsfc.nasa.gov/index.html.

${ }^{2}$ See http://www.helio-vo.eu.
} 
Sun, and at $62^{\circ}$ heliographic latitude and $116^{\circ}$ longitude east of the Earth. The authors report large fluxes of SEPs at energies $>100 \mathrm{MeV}$ at both ULYSSES and the Earth, and that the particles observed at ULYSSES were recorded at greater intensities than expected if they were transported along magnetic field lines alone. They conclude that cross-field transport was likely to be involved, otherwise ULYSSES would not have observed the particles.

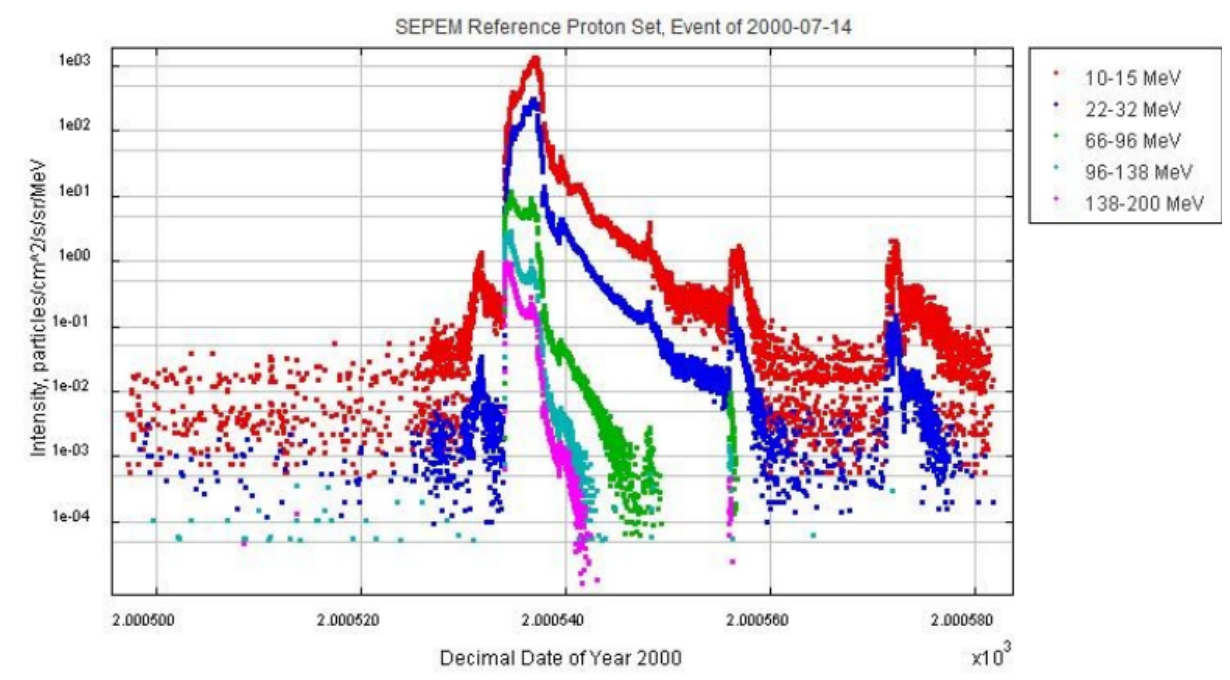

Figure 5. 2000-07-14 SEP Event Time-intensity Profile In this image, the intensities of protons are shown for the energy channels 10-15 MeV (red), 22-32 MeV (blue), 66-95 MeV (green), 96-138 MeV (cyan), 138-200 (pink) for the month of July 2000.

The expanded profile of the Bastille day event is shown in Figure 6 for the satellites GOES (panel a)), SOHO EPHIN (panel b)), SOHO ERNE (panel c)), and WIND (panel $\mathrm{d}))$ covering a period of $\approx 4.5$ days. In this profile, there is a shock peak at around 13:05 on the 15th of July, after which there is a gradual decay at all energies from 10 to $200 \mathrm{MeV}$. GOES proton fluxes reach $10^{3}$ particles $\mathrm{cm}^{-2} \mathrm{~s}^{-1} \mathrm{sr}^{-1}(\mathrm{MeV} / \mathrm{nuc})^{-1}$ at $10-15 \mathrm{MeV}$, and 1 at $138-200 \mathrm{MeV}$, which is a very strong particle event. SOHO ERNE protons reach a peak flux of $10^{2}$ in the 8.1-10 MeV channel, whereas the EPHIN instrument protons reach what could be a flux of $10^{3}$ flux at lower energy channels from 4.3-7.8 MeV (the instrument was blinded during the onset of the event). Like IMP 8, WIND was placed in a halo orbit at L1, and should have similar fluxes to GOES and SOHO, except for the fact that in 2000 NASA moved WIND into various L2 positions $\approx 1.5-2.0$ million miles on the other side of the Earth away from the Sun in the magnetotail. This would explain the much lower fluxes observed in the keV ranges. This flare and its associated CME is not related to a single active region according to Wang et al. (2006), who reported that the activation and eruption of a huge trans-equatorial filament preceded the flare and CME, and that many tens of halo-CMEs are found to be associated with trans-equatorial filaments 
and their magnetic environments. According to the authors, this could shed new light on CME magnetism.

The spectral properties of the Bastille day events have been studied by Bombardieri et al. (2006) using spectral fits to the spacecraft and neutron monitor data. A spectral "knee" was observed by Wang and Wang (2005), which occurred at $>30 \mathrm{MeV}$ over an energy range from 1 to $500 \mathrm{MeV}$. The energy spectra of Fe for this event was very different from other species according to Tylka et al. (2001a,b), which the authors explained is due to a two component source population from the flare and CME. The authors said that the 5 percent admixture of flare remnants has an effect on the event-to-event variability of SEPs in large gradual SEP events, but the predominant accelerator is the CME shock.

The energy spectra of the Bastille Day event of 2000-07-14 are shown in the Figure 7 montage, in a similar manner to the event of 1977-11-22, with each panel in the montage corresponding to the vertical lines delimited in Figure 6 ( 8 hours 46 minutes between lines). The montage gives the spectral plots versus time for ACE ULEIS and SEPICA, GOES 11, IMP8 CRNC and GME, and SOHO ERNE. In the first panel (N0.1.11), the background flux is shown, which would be as in segment No 1 of Figure 6 . As these are all near-Earth spacecraft, the spectra would be expected to exhibit similar behavior. In the next panel, the onset of the flux enhancement of the event one hour later, we see the high energy particles arrive at GOES in the $60-200 \mathrm{MeV} / \mathrm{n}$ energy range, and 50$90 \mathrm{MeV}$ energy range at IMP $8 \mathrm{CRNC}$, and $90-200 \mathrm{MeV} / \mathrm{n}$ at IMP 8 GME. Unfortunately, SOHO ERNE is missing data in this and the remaining panels (apart from the last panel (No 13.1) at the high energies, but no particles are observed at this time at the lower $<10 \mathrm{MeV} / \mathrm{n}$ energy range. One hour later in panel 2.1 , we see a strange increase in the mid-range energies from $10-30 \mathrm{MeV} / \mathrm{n}$ at IMP 8 GME, with no increase at the other energies, creating a "Z" shaped spectra. The higher energy particles of GOES 11 and IMP 8 CRNC continue to increase from the background rate of $10^{-5}-10^{-4}$ to $10^{0}$, with no particles arriving at the lower energies for all spacecraft apart from ACE SEPICA. In the next panel, No 2.2, the higher energy channels of IMP 8 GME are not available, and so we will not know whether the "Z" shape would have persisted. However, we still have the higher energies up to $95 \mathrm{MeV}$ for IMP 8 CRNC, which serve as a proxy for the loss of the GME data. There is very little change compared with panel 2.1 in the spectra for all the spacecraft in this panel which is just 3 hours after the onset. After a further six hours in panel No 2.8, the lower energy particles begin to arrive in the $3-10 \mathrm{MeV} / \mathrm{n}$ range for all spacecraft. IMP8 CRNC shows an "S" shape at the $15-90 \mathrm{MeV} / \mathrm{n}$ energy range as a result of low energy particles arriving at the $10-20 \mathrm{MeV}$ range. At $\approx 29$ hours after the onset of this event, in panel 5.1, there is a huge surge of low energy particles reaching a flux of $6 \times 10^{4}$ at IMP 8 GME, which is the peak flux for the lower energies and is associated with the passage of the interplanetary shock in segment 5 of Figure 6. The remaining three panels 5.6, 6.2 and 13.1 are the decay phase of this event, with the spectra of all spacecraft becoming very similar apart from ACE SEPICA, which returns to a particle flux similar to its observed background levels. Once again, we see the wave-like pattern in the spectra rising up at the higher energies from the onset of the event flowing up to the lower energies, and 
an 'arch' structure in panel 2.8. It has taken $\approx 29$ hours for the lower energy particles to reach peak intensities.

It is clear that the very high particle fluxes during the Bastille day events posed a challenge to many of the instruments. Instrumental effects are the likely cause of the large differences in the spectra measured at the near-Earth spacecraft. 

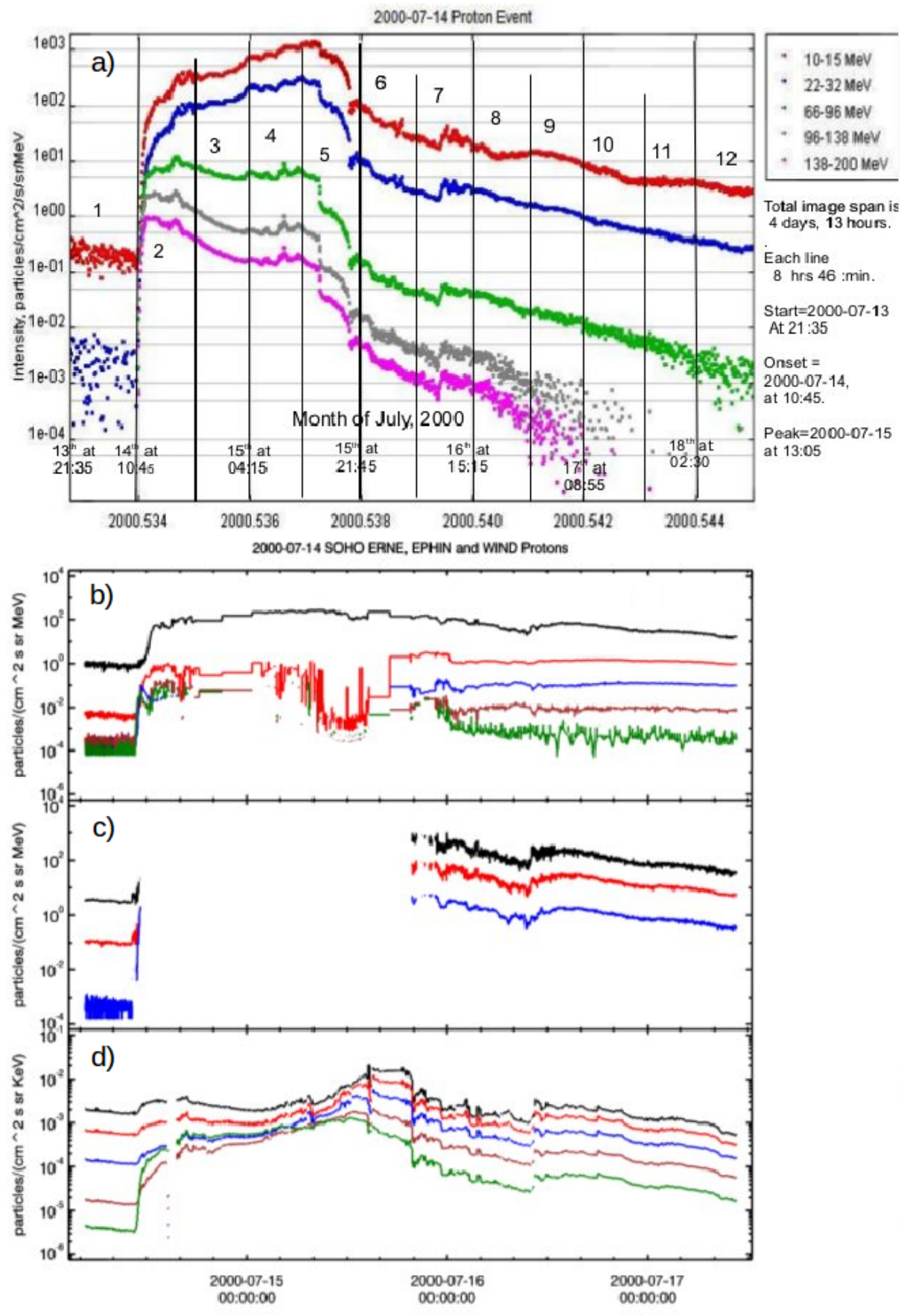

Figure 6. 2000-07-14 SEP Event Time-intensity Profiles as measured by GOES, top panel a) 10-15 MeV (red), 22-32 MeV (blue), 66-95 MeV (green), 96-138 MeV (gray), 138-200 (violet); SOHO EPHIN panel b) 4.3-7.8 MeV (black), 7.8-25 MeV (red), 25-40.9 MeV (blue), 40.9-53 $\mathrm{MeV}$ (brown); SOHO ERNE panel c) 8.1-10 MeV (black), 21-28 MeV (red), 40-51 MeV (blue), 64-80 MeV (brown), 101-131 MeV (green), and WIND panel d) 429-676 keV (black), 676-1360 keV (red), 1360-2787 keV (blue), 2787-6092 keV (brown), 6093-7087 keV (green). 


\section{J. Doran, S. Dalla}
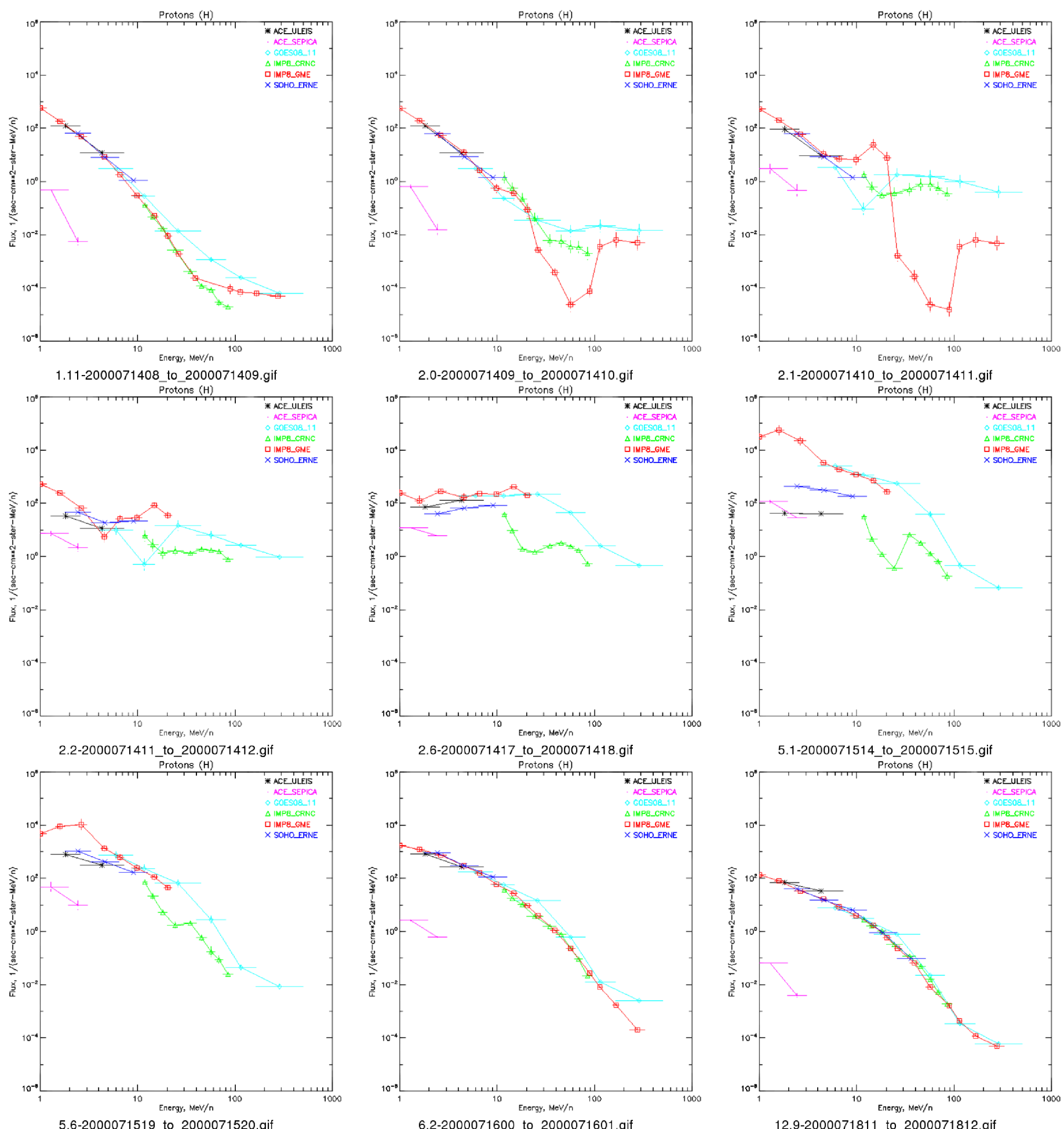

Figure 7. The Time Evolution of The Energy Spectra For The SEP Event of 2000-07-14 measured at multiple spacecraft. The decimal number labeling each plot corresponds to the time interval in Figure 5 a). ACE ULEIS data are in black, SEPICA in pink, GOES 11 in light blue, IMP 8 CRNC in green, GME in red, and SOHO ERNE in blue. 
The event averaged spectrum for ACE, GOES, IMP 8, and SOHO over 4.5 days from 2000-07-14 at 08:00 hours to 2000-07-19 at 19:00 hours is shown in Figure 8 . The spectra at the different locations show significant differences, unlike the event of 1977-11-22. This could be due to the late arrival of the low energy particles in the 1-10 MeV range approximately 34 hours after the higher energy particles in the $10-200 \mathrm{MeV}$ range, which is only 8 hours for the 1977-11-22 event.

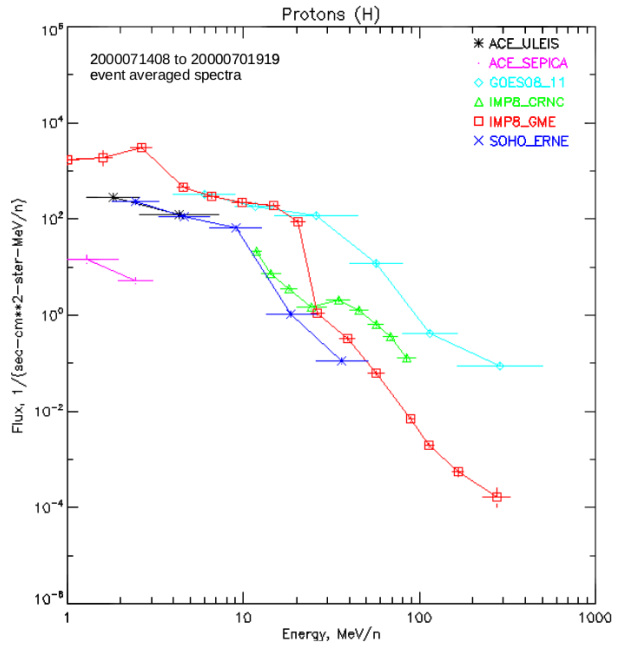

Figure 8. 2000-07-14 to 2000-07-19 Event Averaged Spectra as measured by ACE ULIES (black color), ACE SEPICA (pink), GOES 11 (light blue), IMP 8 CRNC (green), IMP 8 GME (red), and SOHO ERNE (blue).

\subsection{Event of 2001-04-15}

The event of 2001-04-15 took place during a period of intense solar activity which produced at least four SEP events over the space of $\approx 3$ weeks from April 1 , 2001, as shown in Figure 9. The event of interest is the third peak in the figure, which is expanded in Figure 10 covering a period of $\approx 3.63$ days. The CDAW and HELIO catalogs show that these four events are caused by halo CMEs taking place on April 2, 10, 15, and 18, but the CME of interest is the one listed on April 15, 2001.

The International Space Station (ISS) observed two SEP events which were reported by Johnson et al. (2006), during the week of April 15 (there was only one event that week on the 15 th, whereas there was one on the 10 th the previous week, and one on the 18th the following week), which resulted in the exposure of the crew to a record time-resolved dose of radiation. Two events were also reported by Tylka et al. (2002) for the 14th and 15th, the second one of which was thought to be associated with a flare using data from ACE, WIND, and IMP8 covering an energy range of $\approx 30 \mathrm{keV} /$ nuc to $\approx 400 \mathrm{MeV} /$ nuc. They report that these two events were the largest impulsive and ground level events observed 
in cycle 23, having come from the same active region on the Sun into similar IP space conditions. They associate the two distinctly different events with a large western longitude flare and a fast moving CME.

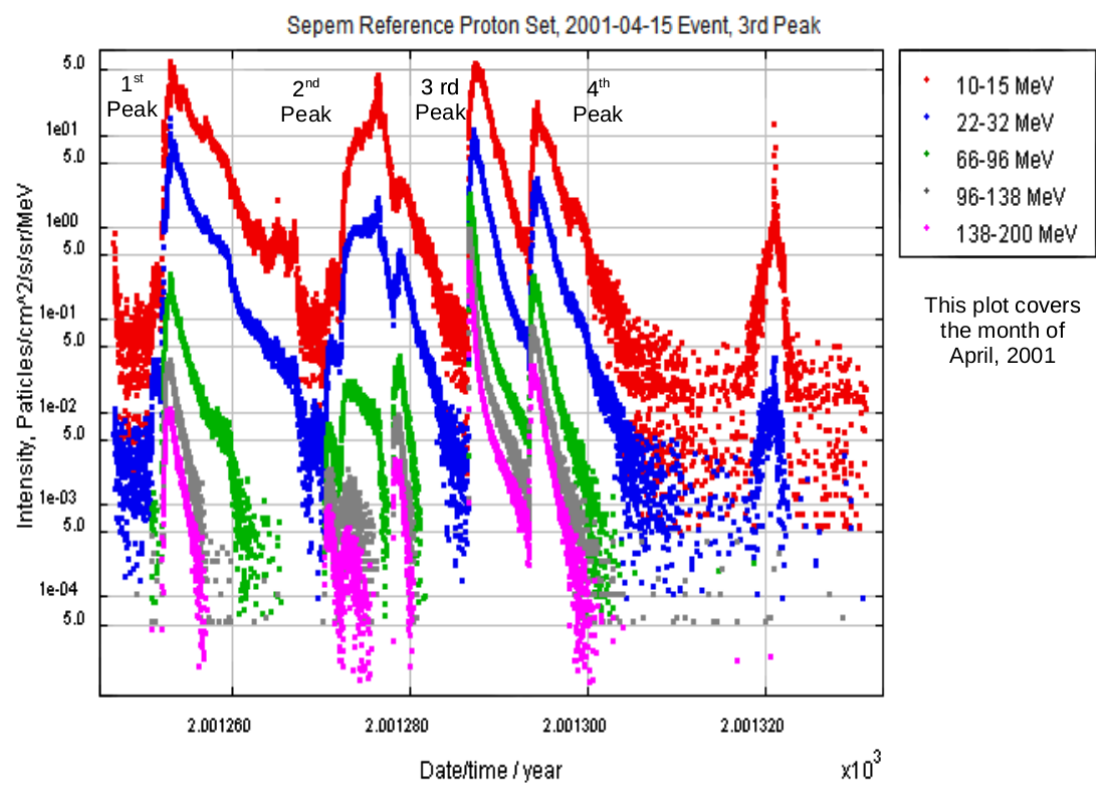

Figure 9. 2001-04-15 SEP Event Time-intensity Profile. In this image, the intensities of protons for $4 \mathrm{SEP}$ events are shown for energy channels 10-15 MeV (red), 22-32 MeV (blue), 66-95 MeV (green), 96-138 MeV (gray), 138-200 (pink) for the month of April, 2001. The event of interest is the third peak, which has a very high particle flux in the $138-200 \mathrm{MeV}$ energy channel.

This view was also supported by Rujiwarodom et al. (2003), who reported that the IP transport of the events was diffusive, and that particle acceleration began at 13:42 UT (when observed as a GLE on neutron monitors) at the peak of the flare emissions, and also at the onset of the shock-related emissions of Type II and IV radio bursts, supporting the view that a CME not a flare was responsible for the relativistic ion acceleration. Further support for view of a CME/flare combination comes from Gopalswamy et al. (2003), who compared the two events of April 14th and 15th. The authors reported that the weak event of the 14th was associated with a $830 \mathrm{~km} / \mathrm{sec} \mathrm{CME}$, while the largest event of cycle 23 on April 15 (the third peak of Figure 9) was associated with a faster $1200 \mathrm{~km} / \mathrm{sec}$ CME and X14.4 flare.

This event was also widely observed at ground level by the neutron monitoring network. Relativistic protons were reported by Muraki et al. (2008) to have resulted in a GLE, while GOES 10 measured proton flux directly. It was also reported by the IPS Radio and Space Service, and described by D'Andrea and Poirier (2003), as a rare X14 flare at 1 AU from the Sun originating from coordinates S20W85. They mentioned that only 16 flares of GOES class $+\mathrm{X} 10$ or larger were reported from the 22 year period between 1976 and 1998. Further 
interesting observations were reported by Maia et al. (2007), on the Nançay radioheliograph, which observed fast-moving loops in images taken in the 164$432 \mathrm{MHz}$ range, tracking them from 0.1-2.5 solar radii above the limb of the Sun. They also report white-light images from the Large Angle Spectrometric Coronagraph (LASCO) on board the SOHO spacecraft. In addition, the authors report that the event is well associated with an energetic electron event observed by the Electron, Proton, and Alpha Monitor (EPAM) on board the ACE satellite.

The spectra for this event are shown in the montage in Figure 11, beginning with panel No. 1.4 representing the background flux for this event, which is relatively high flux of $10^{-1}$ for the $10-15 \mathrm{MeV}$ energy channel due to the decay of the previous event masking the onset at the low energies. One hour later in the next panel No. 1.5, we see the onset of the flux enhancement, with the higher energy particles $>10 \mathrm{MeV} / \mathrm{n}$ arriving at all spacecraft (ACE ULEIS/SEPICA, GOES 11, IMP 8 CRNC/GME, and SOHO ERNE). In the next panel No. 2.2 , two hours later, all spacecraft continue to record further increases in the higher energy particles, with little sign of the arrival of the lower energy particles $<10 \mathrm{MeV} / \mathrm{n}$. A wave-like spectral shape is beginning to emerge in panel 3.1, approximately five hours after the onset of the event, with a decrease in the particles recorded at the highest energies while increasing through the mid-range of energies, and with little or no increase at the lowest energies. By panel No. 3.2 one hour later, the wave-like shape and "arch" structure of the spectra is now more pronounced with further increase in flux at the mid-range of energies while remaining constant at the lowest and highest energies. The time of the peak intensity at $\approx 10 \mathrm{MeV}$ for this event is in the fourth segment of panel 4.2, which is 11 hours after the onset of the event, with particles now arriving at the lowest energies. In panel 6.5 the wave-like pattern completes its propagation and the spectrum becomes approximately a power law shape. In the remaining two panels 10.2 and 14.4, we see the decay phase of the event, with spectra becoming very similar to each other and the flux returning close to the background rate before the onset of the next event. All spacecraft are equally well connected to the magnetic field lines as would be expected for their near-Earth positions. Once again, we observe the wave-like pattern of the spectra evolving from the highest energies and flowing up the slopes of the spectral curves to the lowest energies. The delay in the arrival of the lowest energy particles of 11 hours after the onset of the flux enhancement could mean an excess of high energy particles. 

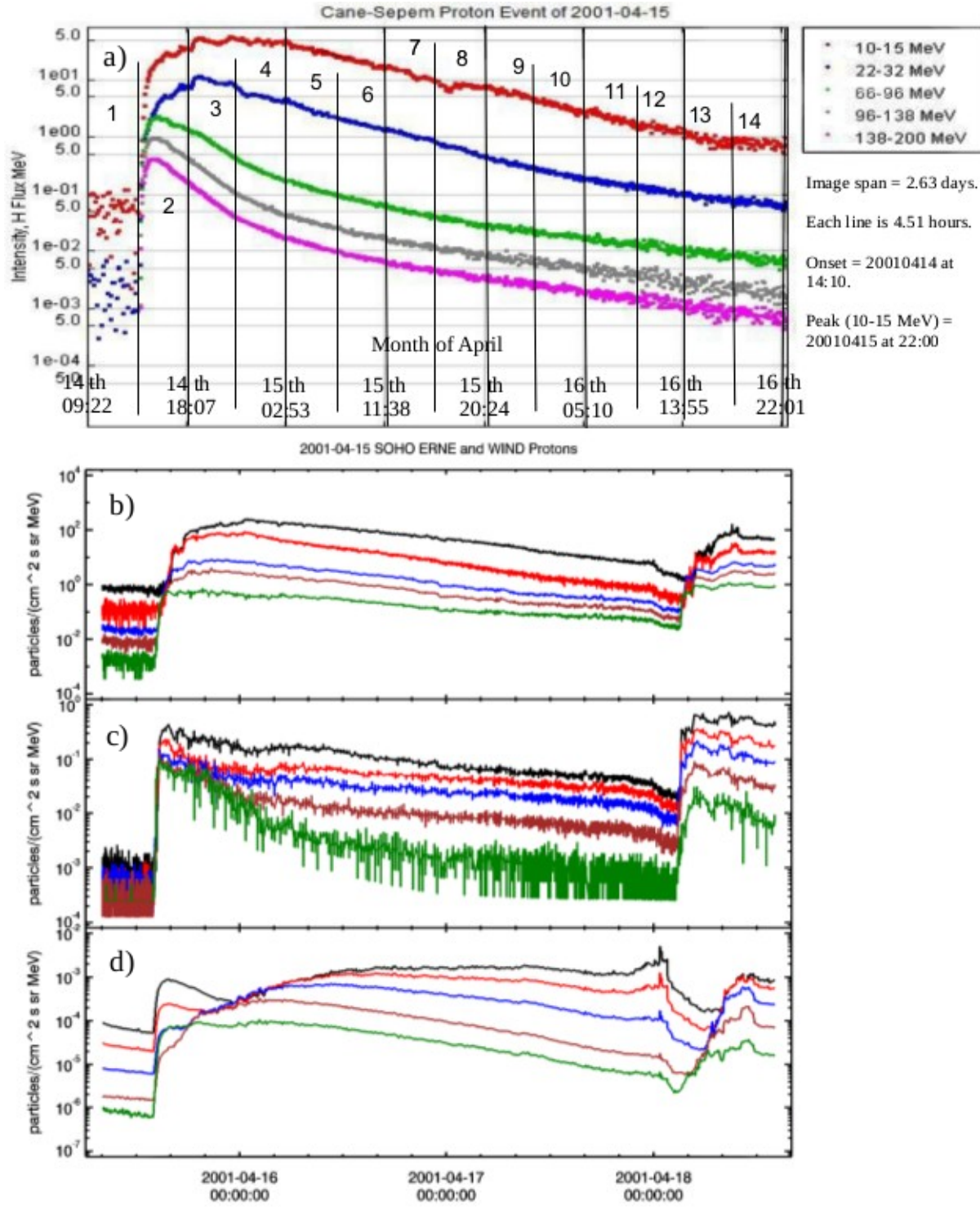

"SEPServer

Figure 10. 2001-04-15 SEP Event Time-intensity Profile as measured by GOES, top panel a) 10-15 MeV (red), 22-32 MeV (blue), 66-95 MeV (green), 96-138 MeV (gray), 138-200 (violet); SOHO ERNE panel b) 4.3-7.8 MeV (black), 10-13 MeV (red), 17-22 MeV (blue), 21-28 MeV (brown), 32-40 MeV (green); SOHO ERNE panel c) 40-51 MeV (black), 51-67 MeV (red), 64-80 $\mathrm{MeV}$ (blue), 80-101 MeV (brown), 101-131 MeV (green); WIND panel d) 429-676 keV (black), $676-1360 \mathrm{keV}$ (red), $1360-2787 \mathrm{keV}$ (blue), 2787-6092 keV (brown), 6093-7087 keV (green). The data of the SOHO and WIND spacecraft extend into fourth peak of Figure 9 or $\approx 1.5$ days longer time than for the GOES data. The small peak in panel d) at about 2001-04-18 00:00:00, could be attributed to energetic storm particles (ESPs) according to Huttunen-Heikinmaa and Valtonen (2009), since the intensity enhancements at several energy channels take place with no velocity dispersion in the vicinity of a shock passage. 

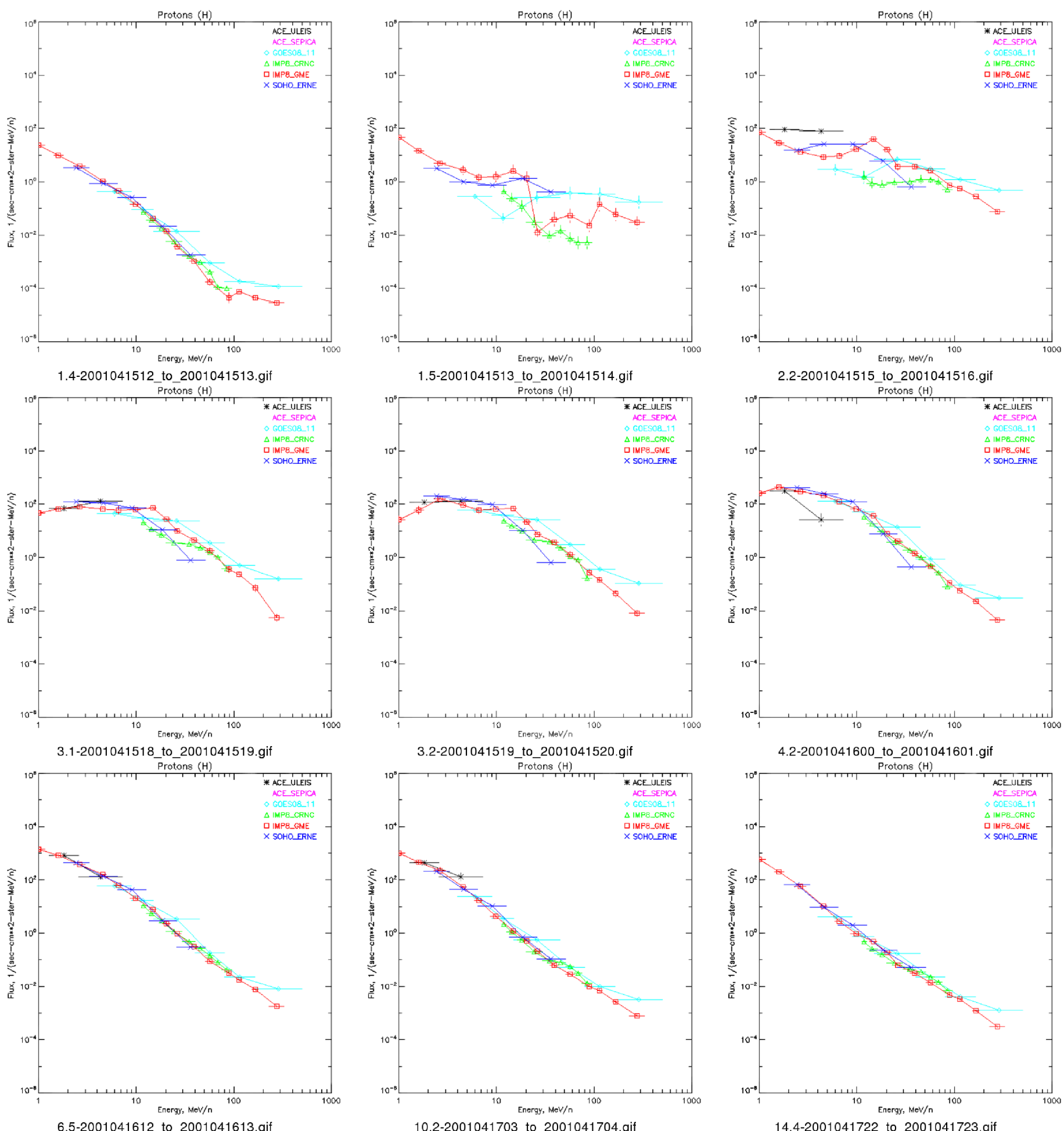

Figure 11. Time Evolution of The 2001-04-15 Event Energy Spectra measured at multiple spacecraft. The decimal number labeling each plot corresponds to the time interval in Figure 10. ACE ULEIS data are in black, SEPICA in pink, GOES 11 in light blue, IMP 8 CRNC in green, GME in red, and SOHO ERNE in blue. 
The event-averaged spectrum for ACE, GOES, IMP 8, and SOHO over 5.5 days from 2000-07-14 at 08:00 hours to 2000-07-19 at 19:00 hours is shown in Figure 12, where the spectra are all very similar in a power law shape over the energy range from 1 to $300 \mathrm{MeV}$. This spectrum resembles the event of 1977-1122 in Figure 4, where the spectrum is very similar but harder compared with the spectral snapshots later in that event. In this event, the lower-energy particles arrive only 6 hours after the higher energy particles, compared to 8 hours for the 1977-11-22 event.

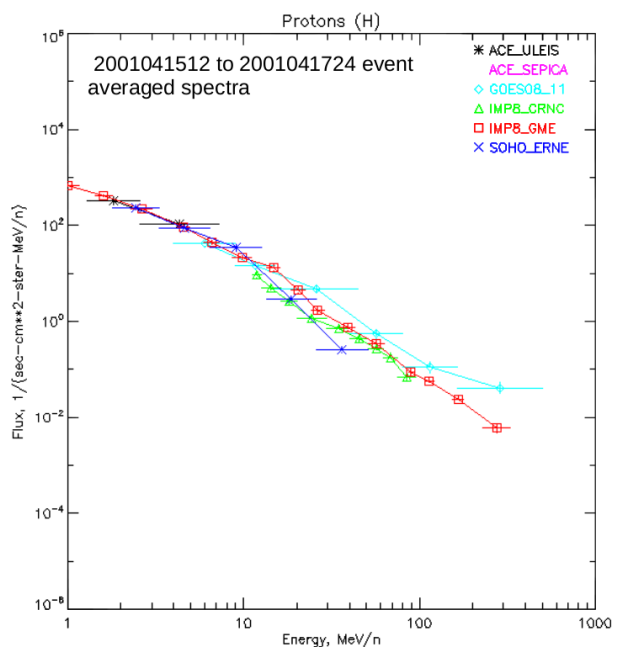

Figure 12. 2001041512 to 2001041524 Event Averaged Spectra as measured by ACE ULIES (black color), ACE SEPICA (pink), GOES 11 (light blue), IMP 8 CRNC (green), IMP 8 GME (red), and SOHO ERNE (blue).

\subsection{Event of 2012-03-07}

This is a STEREO-era SEP event, which is associated with a very active solar phase at NOAA region 11429. Liu et al. (2014) reported a series of flares and CMEs producing a cluster of shocks and transient streams that interacted with one another resulting in a large merged interaction region (MIR) with a preceding shock. Details from the CDAW and HELIO catalogs show that the SEP event was associated with a halo CME at N22E12 with a velocity of $2684 \mathrm{~km} / \mathrm{sec}$ beginning at 00:24, and with an associated flare of X5.4 class from the same active region (possibly at N17E15) following at 02:00. The positions and orbital trajectories of the STEREO A and B spacecraft relative to near-Earth and the flare direction on the Sun are shown in Figure 13, where the separation between the two STEREO spacecraft is $132.7^{\circ}$.

The time-intensity profile for the GOES 13 near-Earth spacecraft is shown in Figure 14 as peak A, beginning at a relatively high background flux of $4 \times 10^{-1}$ particles $\mathrm{cm}^{-2} \mathrm{~s}^{-1} \mathrm{sr}^{-1}(\mathrm{MeV} / \mathrm{nuc})^{-1}$ in 10 to $15 \mathrm{MeV}$ channel. The event shows a strong flux of 600 particles $\mathrm{cm}^{-2} \mathrm{~s}^{-1} \mathrm{sr}^{-1}(\mathrm{MeV} / \mathrm{nuc})^{-1}$ in the $10-15 \mathrm{MeV}$ energy channel, and 0.2 particles in the $138-200 \mathrm{MeV}$ energy channel. The small 


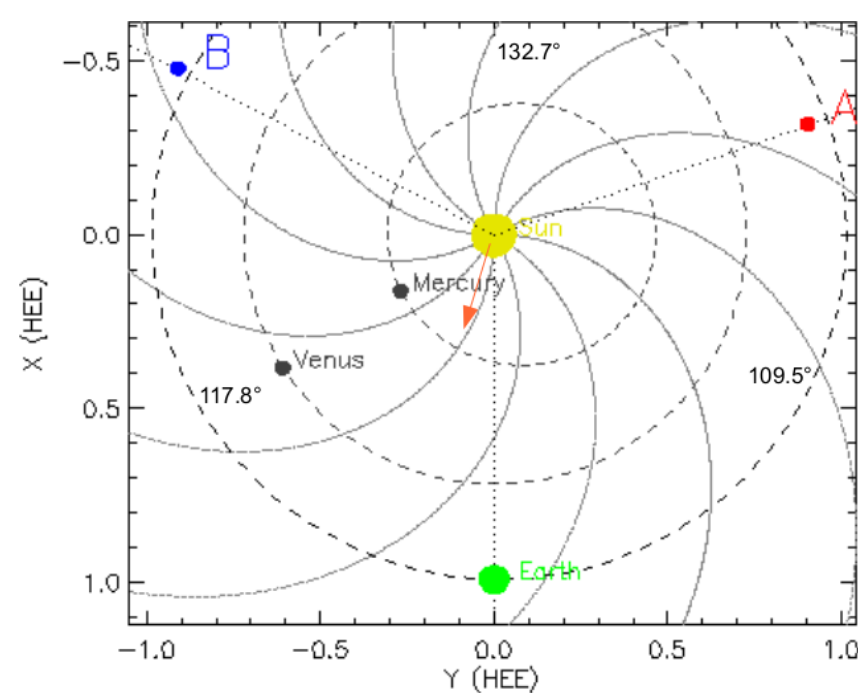

Figure 13. Positions and Orbital Trajectories of STEREO A and B during SEP event of 20012-03-07. In the figure, the $\mathrm{Y}(\mathrm{HEE})$ and $\mathrm{X}(\mathrm{HEE})$ axis are the Heliocentric Earth Ecliptic along the $X$ and $Y$ axis of the Earth-Sun line, and the $Z$ axis is the ecliptic north pole. The STEREO A (ahead of the Earth) spacecraft is the red circle at 0.957136 AU, the Earth's orbit is the dashed circle, and the STEREO B (behind the Earth) spacecraft is the blue circle at $1.02973 \mathrm{AU}$. The Earth is shown as the green circle, and the orange arrow is the position and direction of the flare. The separation angle with Earth for STEREO A is $109.5^{\circ}$, and for STEREO B is $117.8^{\circ}$, with the separation angle with A and B being $132.7^{\circ}$. Light travel times to Earth are $13.2 \mathrm{~min}$ for A and $14.4 \mathrm{~min}$ for B.

hump prior to peak $A$ is reported by the SOHO LASCO CME catalog as being associated with two small $C$ to $M$ class events at N16E30 and N19E32 respectively. The expanded profile for this event is shown in Figure 15, giving profiles for GOES 13 panel a), STEREO A HET panel b), and STEREO B HET panel c). The time axis for the STEREO A and B plots are synchronized with the GOES plot, so that the segmented lines in the GOES plot apply to them as well. These numbered segments refer to the numbered panels of the spectra in Figure 17. The time-intensity profiles show that STEREO A is not as well connected to the magnetic field lines, but the profile is recognizable and similar to GOES, whereas STEREO B observes a strong flux of between 5 and 100 particles $\mathrm{cm}^{-2} \mathrm{~s}^{-1} \mathrm{sr}^{-1}(\mathrm{MeV} / \mathrm{nuc})^{-1}$ in the 60 to $100 \mathrm{MeV}$ and 13.6 to 15.1 MeV energy channels respectively. The lower energy profiles for STEREO A and B using the LET instrument are shown in Figure 16, where again STEREO $\mathrm{A}$ is not well connected to the magnetic field lines, but STEREO B registers very high flux between 200 and 1000 particles $\mathrm{cm}^{-2} \mathrm{~s}^{-1} \mathrm{sr}^{-1}(\mathrm{MeV} / \mathrm{nuc})^{-1}$ in the $10-12 \mathrm{MeV}$ and 4.0-4.5 MeV energy channels respectively.

The event of 2012-03-07 is an interesting multi-spacecraft observed SEP event related to a series of CMEs and flares from the same active region on the Sun. It was well observed by GOES 13, SOHO ERNE, STEREO B (and to a lesser extent, STEREO A), and also the ACE low energy channels. It has a shock associated sharp spike in the flux at the lower energy channels of 10-15 


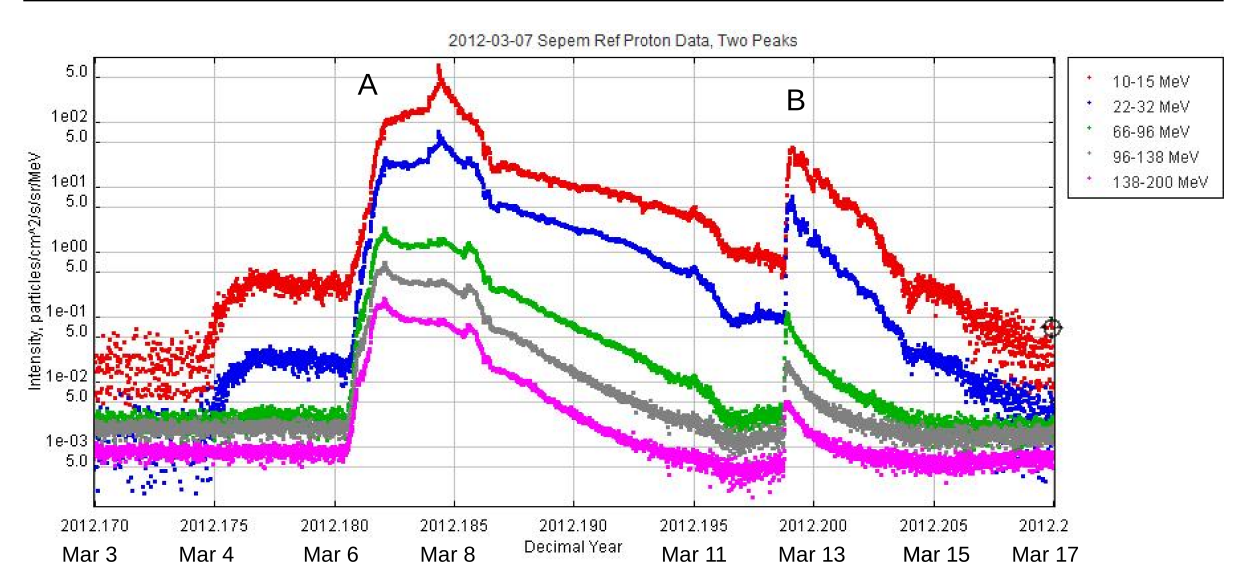

Figure 14. 2012-03-07 Time-intensity Profile. This image is a near-Earth plot of the SEPEM GOES data for the intensities of protons for two SEP events for energy channels 10-15 MeV (red), 22-32 MeV (blue), 66-95 MeV (green), 96-138 MeV (gray), and 138-200 (pink) for the month of March 2012. The event of interest is the peak A, which has a sharp shock spike at the lower energies (red and blue plots) at 2012-03-08 at 11:15.

$\mathrm{MeV} / \mathrm{n}$ and $22-32 \mathrm{MeV} / \mathrm{n}$, but the remaining higher energy channels are close to the classic profile for an SEP event.

A montage of images for the evolution of the spectra versus time is shown in Figure 17, where the panels are numbered according to the vertical segments in the profile view of Figure 15. Beginning with panel 1.1, segment 1, the spectra of the background flux is shown. One hour later in the next panel 1.2, segment 1 , is the beginning of the onset of the flux enhancement of the event, where STEREO B HET is the first to observe any particles, at the higher energies. In panel 1.3, GOES 13 begins to observe particles in the 138-200 MeV energy channels, while the STEREO B flux continues to increase. As yet there is no response from SOHO, STEREO A, or ACE. In panel 1.6 (six hours after the onset and $\approx 1 / 3$ along segment 1 ), we see a large increase in flux in GOES 13 at the higher energies from 10-200 MeV, and SOHO and STEREO B now begin to observe particles at the $>10 \mathrm{MeV} / \mathrm{n}$ energies. The ACE spacecraft also records a small increase in flux at the lower energies of $2-5 \mathrm{MeV} / \mathrm{n}$. The lower energy particles now begin to arrive in panel 1.14 (14 hours after the onset, $\approx 3 / 4$ along segment 1), for all spacecraft, and GOES 13 now records more particle flux than STEREO B, 13 hours after the onset of the event. After $\approx 4$ hours in panel 2.1, segment 2, SOHO observes a drop in the higher energies and an increase in the lower energy particles steepening the slope of the curve, as if the energy was being transferred from one to the other. In the next panel $3.4(\approx 36$ hours after the onset) the effect of the steepening of the slope of the GOES 13 spectral curve is even more pronounced. This panel is at the time of maximum flux for the lower energy particles between $10^{3}$ and $10^{4}$ particles $\mathrm{cm}^{-2} \mathrm{~s}^{-1} \mathrm{sr}^{-1}(\mathrm{MeV} / \mathrm{nuc})^{-1}, \approx \mathbf{3 4}$ hours after the event is in progress, with the spectra at all spacecraft becoming invariant. GOES 13 and SOHO at this point have a sort of "knee" shape in the 20-40 MeV range, with the decay of these particles lagging behind the higher 

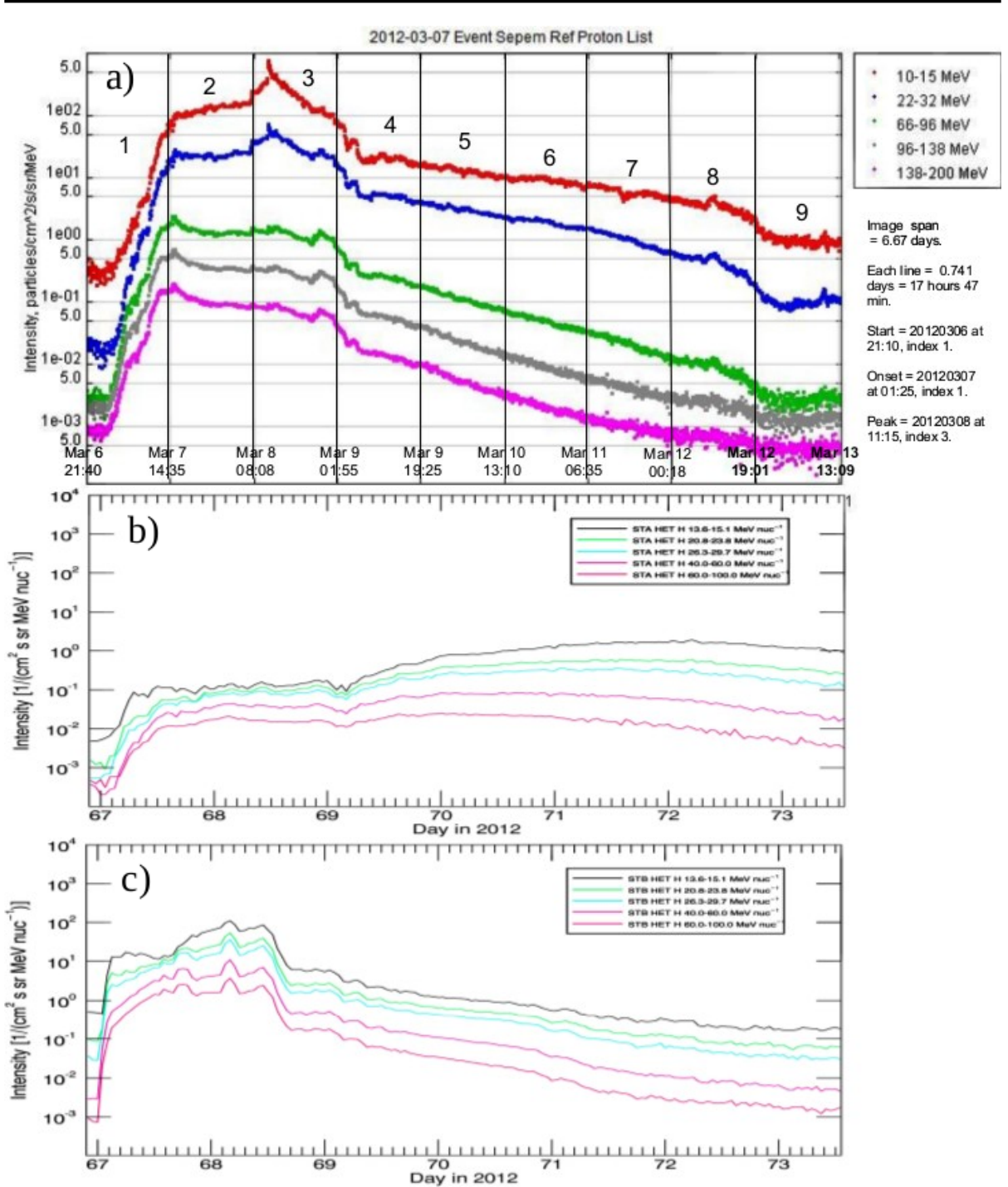

Figure 15. 2012-03-07 Time-Intensity Profiles for GOES, top panel a) 10-15 MeV/n (red), $22-32 \mathrm{MeV} / \mathrm{n}$ (blue), 66-95 MeV/n (green), 96-138 MeV/n (gray), 138-200 MeV/n (violet); STEREO A HET panel b) $13.6-15.1 \mathrm{MeV} / \mathrm{n}$ (black), $14.9-29.1 \mathrm{MeV} / \mathrm{n}$ (5 green lines), 29.5-33.4 $\mathrm{MeV} / \mathrm{n}$ (blue), 33.4-35.8 MeV/n (light blue), 35.5-40.5 MeV/n (1st pink), 40-60 MeV/n (2nd pink), $60-100 \mathrm{MeV} / \mathrm{n}$ (last pink line); STEREO B HET panel c), plotted in similar colors and energy channels as STEREO A HET.

energies. By panel 9.1, approximately 140 hours after the onset, the flux has decayed to the background level. As mentioned above, the background flux is relatively high at $4 \times 10^{-1}$ particles $\mathrm{cm}^{-2} \mathrm{~s}^{-1} \mathrm{sr}^{-1}(\mathrm{MeV} / \mathrm{nuc})^{-1}$, because this event is only one of several CMEs and flares from this active region on the Sun. Nevertheless, there is a distinct wave-like pattern and "arch" structure in the 

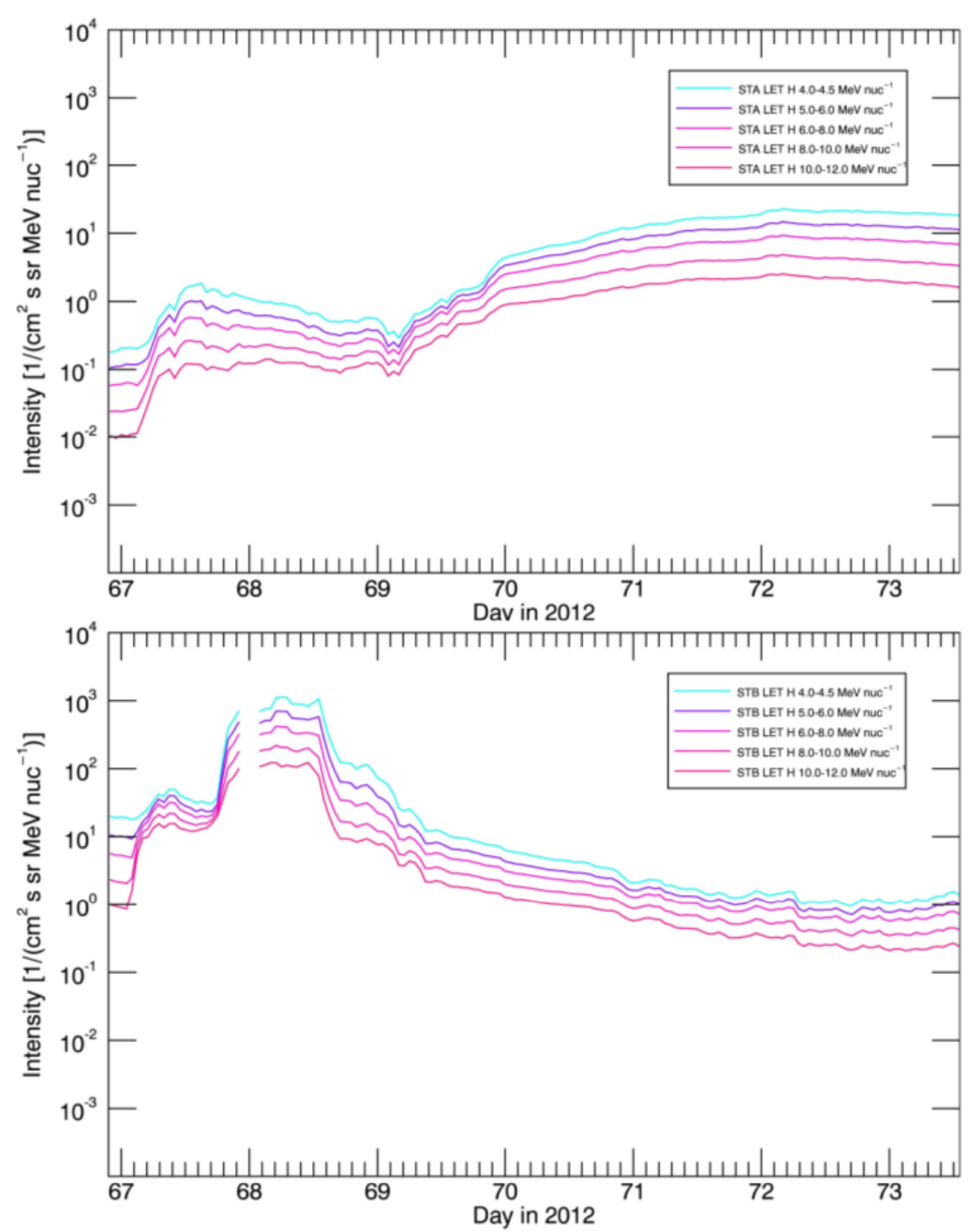

Figure 16. 2012-03-07 Time-Intensity Profiles for STEREO A LET (top panel) and STEREO B LET (bottom panel) at energy channels 4.0-4.5 MeV/n (lt.blue), 5.0-6.0 MeV/n (violet), 6.0-8.0 MeV/n (1st pink), 8.0-10.0 MeV/n (2nd pink), and 10.0-12.0 MeV/n (last pink).

evolution of the spectra in a similar manner to those events observed above, and a delay of 14 hours in the arrival of the lower energy particles. 

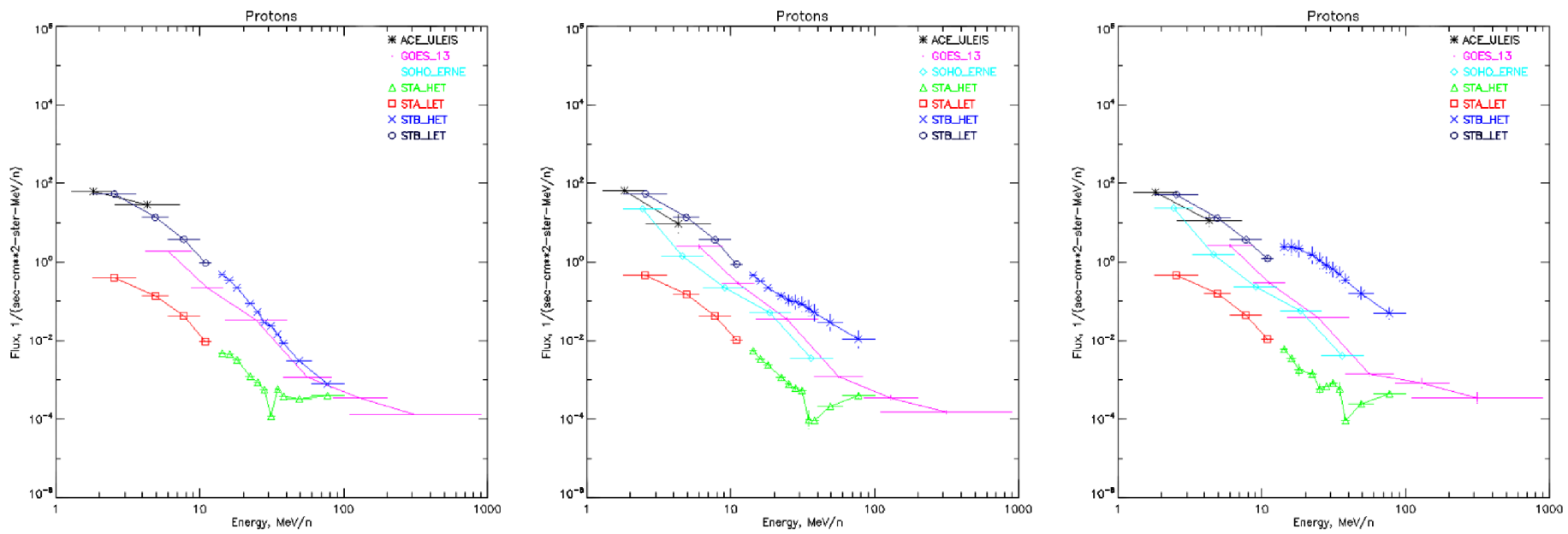

$1.1-2012030623$ to 2012030624 .git
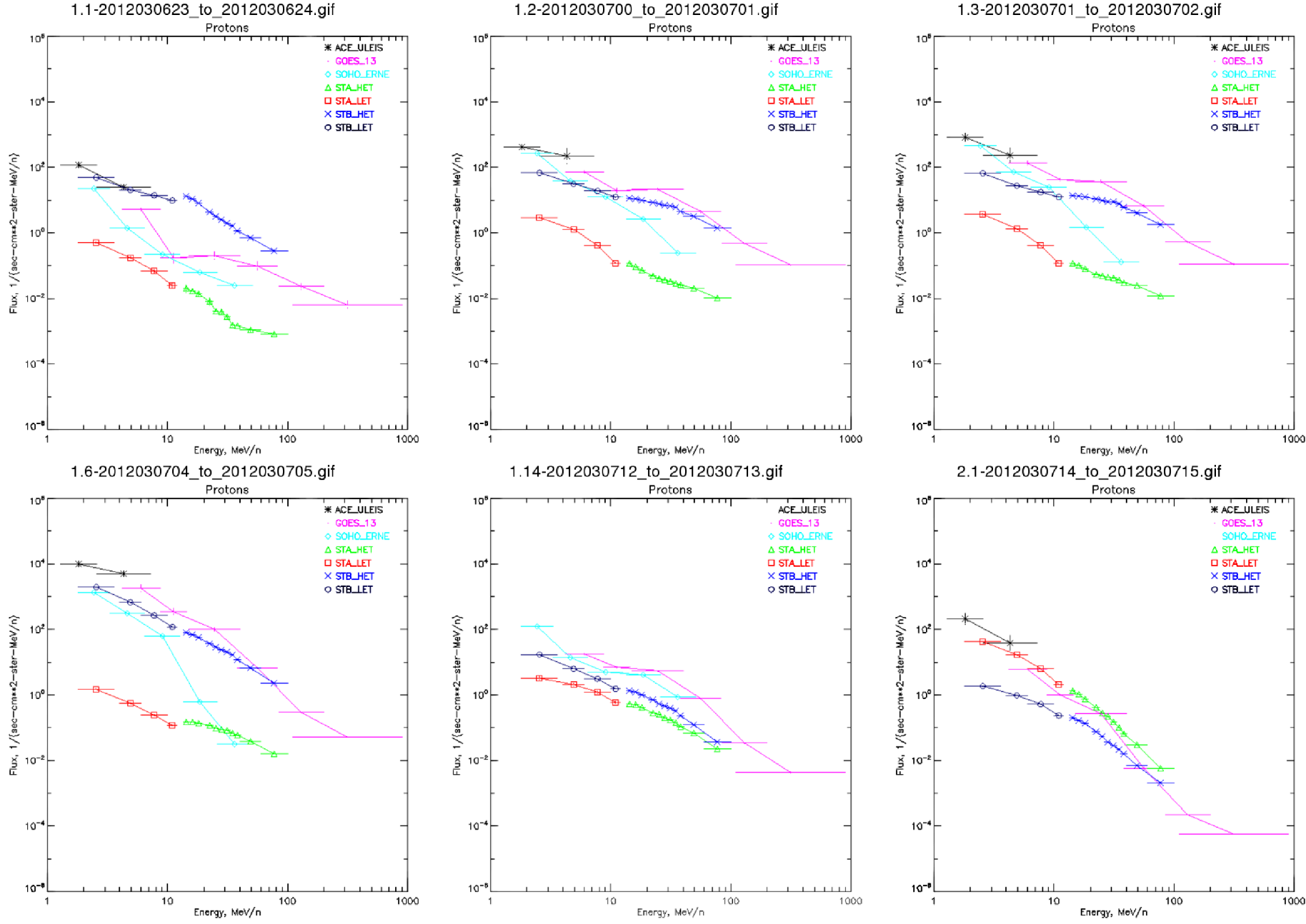

3.4-2012030810_to_2012030811.gif

5.4-2012030920_to_2012030921.gif

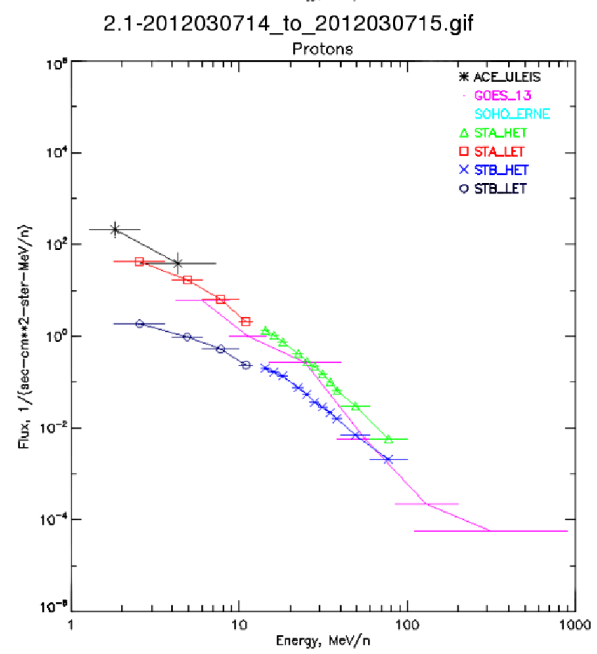

9.1-2012031218_to_2012031219.gif

Figure 17. Time evolution of the energy spectra for event of 2012-03-07 for the 5 spacecraft: ACE ULEIS at 0.6-8.7 MeV/n (black), GOES 11 at $0.6-500 \mathrm{MeV} / \mathrm{n}$ (pink), SOHO ERNE at 1.8-50.7 MeV/n (light blue); STEREO A HET at 13.6-100 MeV/n (light green); STEREO A LET at $1.8-15.0 \mathrm{MeV} / \mathrm{n}$ (red); STEREO B HET at 13.6-100 MeV/n (blue); and STEREO B LET at 1.8-15.0 MeV/n (dark gray). The numbered panels correspond to the numbered segments shown in the profiles of Figure 15, and the time frame for each panel is in bold numerals under each panel. 
The event averaged spectrum for ACE, GOES, SOHO, and STEREO A and B, over 6 days from 2012-03-06 at 23:00 hours to 2012-03-12 at 20:00 hours is shown in Figure 18, where the spectra all display a power law dependence, but show significant differences in the absolute values. These differences could be the result of the wide separation of the STEREO spacecraft away from near Earth locations.

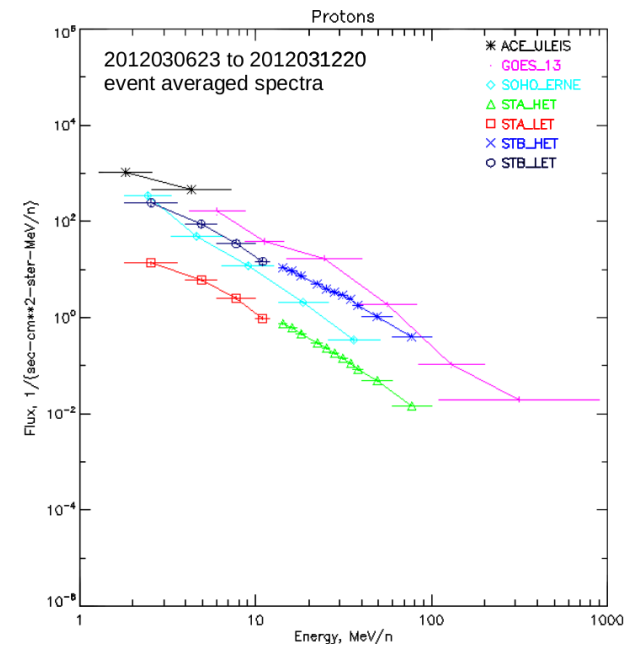

Figure 18. 2012-03-07 to 2012-03-13 Event Averaged Spectra as measured by ACE ULIES (black color, GOES 13 (pink), SOHO ERNE (light blue), STA HET (green), STA LET (red), STB HET (blue), and STB LET (purple)

\subsection{Spectral Index Comparisons}

It is interesting to compare the spectral index for high energies above $40 \mathrm{MeV}$ of the event averaged spectrum with the spectral index of a snapshot of the spectrum late in the event as shown in Table 3 . The values given here show that the event averaged spectra at high energies are generally harder than those late in the event. Late in the event here refers to times when the arch-like structure previously discussed is no longer present in the spectrum, typically $\approx 20-43$ hours after the start of the event. The event integrated spectra for events of 2000-0714 and 2001-04-15 in the table were presented by Mewaldt et al. (2012) in the energy range of 0.1 to $700 \mathrm{MeV}$. They were reported to have a double power-law form with break energies of 24 and $16 \mathrm{MeV}$ respectively.

\section{Discussion and conclusions}

In this paper we presented a detailed analysis of the time evolution of the SEP spectra in four large events associated with major solar eruptive events.

Some of the common features observed in the data we presented are as follows. 
Table 3. The power law index at $>40 \mathrm{MeV}$ late in the event compared to that of the event averaged spectrum for each SEP event.

\begin{tabular}{|c|c|c|c|}
\hline $\begin{array}{l}\text { Event } \\
\text { Date }\end{array}$ & $\begin{array}{l}\text { Spacecraft } \\
\text { Instruments }\end{array}$ & $\begin{array}{l}\text { Power-law index } \\
\text { After the knee }\end{array}$ & $\begin{array}{l}\text { Power-law index } \\
\text { Event averaged }\end{array}$ \\
\hline \multirow{6}{*}{ 1977-11-22 } & HELIOS 1 E6 & -2.84 & -1.97 \\
\hline & HELIOS 1 E7 & -2.71 & -2.13 \\
\hline & HELIOS 2 E6 & -3.67 & -2.31 \\
\hline & HELIOS 2 E7 & -3.20 & -2.12 \\
\hline & IMP 8 CRNC & -2.83 & -2.28 \\
\hline & IMP 8 GME & -2.79 & -2.31 \\
\hline \multirow[t]{6}{*}{ 2000-07-14 } & ACE ULEIS & -1.32 & -0.93 \\
\hline & ACE SEPICA & -3.18 & -1.61 \\
\hline & GOES 11 & -3.12 & -2.28 \\
\hline & IMP 8 CRNC & -3.45 & -2.11 \\
\hline & IMP 8 GME & -2.89 & -3.30 \\
\hline & SOHO ERNE & -2.67 & -2.91 \\
\hline \multirow[t]{5}{*}{ 2001-04-15 } & GOES 11 & -1.78 & -1.28 \\
\hline & ACE SEPICA & -2.39 & -1.90 \\
\hline & IMP 8 CRNC & -2.55 & -2.27 \\
\hline & IMP 8 GME & -2.61 & -2.15 \\
\hline & SOHO ERNE & -2.76 & -2.52 \\
\hline \multirow[t]{7}{*}{ 2012-03-07 } & ACE ULEIS & -0.98 & -0.93 \\
\hline & GOES 13 & -2.61 & -2.33 \\
\hline & SOHO ERNE & -1.87 & -2.50 \\
\hline & Sta HET & -2.57 & -2.31 \\
\hline & Sta LET & -1.77 & -1.77 \\
\hline & Stb HET & -2.32 & -1.95 \\
\hline & Stb LET & -1.47 & -1.91 \\
\hline
\end{tabular}

- Often there are large differences in the measured spectra at the beginning of the events. In most cases these differences are due to the fact that the spacecraft are at large distances from each other in interplanetary space. However, often there are discrepancies in the spectra for spacecraft that are relatively close to each other (e.g. near-Earth spacecraft, see Figure 7 ). This points towards instrumental effects playing a big role, especially during very large SEP events, as well as possibly to the influence of local solar wind effects and of the instrument's viewing direction.

- The wave-like pattern we described in our analysis of the measured spectra, propagating from the high energies to the low energies, is a common feature of the observations. As the pattern propagates through the spectrum, the latter displays an 'arch' shape, which straightens into a power law later in the event. The typical time scale for the propagation of the wave from the high to the low energies is between 9 and 29 hours from the start of the event. 
- The decay phase is characterized by power-law spectra which tend to become similar at far apart locations (cf. invariant spectra, see Reames, Kahler, and $\mathrm{Ng}(1997))$.

- Event integrated spectra are dominated by the late-time intensities at the low SEP energies and by the early time intensities at the high energies.

Several processes are expected to combine to determine the time evolution of the energy spectra as follows.

- Energy and time dependence of the particle release at the source, depending on the acceleration process.

- Particle propagation from the source to the detecting spacecraft, which is influenced by scattering processes and other phenomena that may cause transport (e.g. drifts, field line meandering.)

- Deceleration of particles during their propagation, as a result of adiabatic deceleration e.g. (Ruffolo, 1995) and drift induced deceleration (Kóta, 1979; Dalla, Marsh, and Laitinen, 2015).

Our analysis of the time evolution of the spectra does not enable us to separate the contribution of the above processes to the features of the spectra and their time variation. Modeling is required for this purpose, and we plan to carry out this type of study in the future.

Acknowledgements We thank the instrument teams for providing the data used in this study. We acknowledge use of the VEPO analysis tool as well as data and services provided by SEPEM and SEPSERVER. S.D. acknowledges support from STFC grant ST/M00760X/1. D.D. thanks P. Zelina, University of Central Lancashire, for the use in this study of STEREO plots made with IDL. 


\section{References}

Belov, A.V., Bieber, J.W., Eroshenko, E.A., Evenson, P., Gvozdevsky, B.B., Pchelkin, V.V., Pyle, R., Vashenyuk, V.E., Yanke, V.G.: 2001, The "Bastille Day"; GLE 14 July, 2000 as observed by the worldwide neutron monitor network. International Cosmic Ray Conference 8, 3446. ADS.

Bieber, J.W., Dröge, W., Evenson, P.A., Pyle, R., Ruffolo, D., Pinsook, U., Tooprakai, P., Rujiwarodom, M., Khumlumlert, T., Krucker, S.: 2002, Energetic Particle Observations during the 2000 July 14 Solar Event. ASTROPHYS J 567, 622. DOI. ADS.

Bombardieri, D.J., Duldig, M.L., Michael, K.J., Humble, J.E.: 2006, Relativistic Proton Production during the 2000 July 14 Solar Event: The Case for Multiple Source Mechanisms. ASTROPHYS J 644, 565. DOI. ADS.

Burlaga, L., Lepping, R., Weber, R., Armstrong, T., Goodrich, C., Sullivan, J., Gurnett, D., Kellogg, P., Keppler, E., Mariani, F.: 1980, Interplanetary particles and fields, November 22 to December 6, 1977 - Helios, Voyager and Imp observations between 0.6 and 1.6 AU. J GEOPHYS RES 85, 2227. DOI. ADS.

Cane, H.V., Reames, D.V., von Rosenvinge, T.T.: 1988, The role of interplanetary shocks in the longitude distribution of solar energetic particles. J GEOPHYS RES 93, 9555. DOI. ADS.

Chambon, G., Hurley, K., Niel, M., Talon, R., Vedrenne, G., Estulin, I.V., Likin, O.B.: 1981, The November 22, 1977 solar flare - Evidence for 2.23 and $4.43 \mathrm{MeV}$ line emission from the Signe 2MP experiment. SOL PHYS 69, 147. DOI. ADS.

Crosby, N., Heynderickx, D., Jiggens, P.: 2013, ESA's Solar Energetic Particle Environment Modelling (SEPEM) application server, a www interface to solar energetic particle data. ESA Contract No: 20162/06/NL/JD, Sepem reference proton data set. http://dev.sepem. oma.be.

Crosby, N., Heynderickx, D., Jiggens, P., Aran, A., Sanahuja, B., Truscott, P., Lei, F., Jacobs, C., Poedts, S., Gabriel, S., Sandberg, I., Glover, A., Hilgers, A.: 2015, Sepem: A tool for statistical modeling the solar energetic particle environment. Space Weather 13(7), 406. 2013SW001008. DOI. http://dx.doi.org/10.1002/2013SW001008.

Dalla, S., Agueda, N.: 2010, Role of latitude of source region in Solar Energetic Particle events. Twelfth International Solar Wind Conference 1216, 613. DOI. ADS.

Dalla, S., Marsh, M.S., Laitinen, T.: 2015, Drift-induced Deceleration of Solar Energetic Particles. ASTROPHYS J 808, 62. DOI. ADS.

Dalla, S., Marsh, M.S., Kelly, J., Laitinen, T.: 2013, Solar energetic particle drifts in the Parker spiral. J GEOPHYS RES 118(10), 5979. DOI. http://onlinelibrary.wiley.com/doi/10.1002/ jgra.50589/abstract.

D'Andrea, C.P., Poirier, J.: 2003, A Study of the Ground Level Event of April 15, 2001 with GRAND. International Cosmic Ray Conference 6, 3423. ADS.

Dresing, N., Gómez-Herrero, R., Heber, B., Klassen, A., Malandraki, O., Dröge, W., Kartavykh, Y.: 2014, Statistical survey of widely spread out solar electron events observed with STEREO and ACE with special attention to anisotropies. ASTRON ASTROPHYS 567, A27. DOI. ADS.

Fenton, A.G., Fenton, K.B., Humble, J.E.: 1978, Solar flare increase of cosmic ray intensity on November 22, 1977. P ASTRON SOC AUST 3, 238. ADS.

Gombosi, I.T., Kecskeméty, N., Merényl, L., Táirallyay, M., Kurt, V.G., Logachev, Y.I., Stolpovskii, V.G., Trebukhovskaya, G.A.: 1979, Propagation of Energetic Particles during the November 22, 1977 Event. International Cosmic Ray Conference 5, 163. ADS.

Gopalswamy, N., Yashiro, S., Kaiser, M.L., Howard, R.A.: 2003, Coronal mass ejection interaction and particle acceleration during the 2001 April 1415 events. ADV SPACE RES 32, 2613. DOI. ADS.

Huttunen-Heikinmaa, K., Valtonen, E.: 2009, Interplanetary fast forward shocks and energetic storm particle events above $1.5 \mathrm{MeV}$. Annales Geophysicae 27, 767. DOI. ADS.

Johnson, A.S., Golightly, M.J., Lin, T., Semones, E.J., Shelfer, T., Weyland, M.D., Zapp, E.N.: 2006, A comparison of measurements and predictions for the April 15 and April 18, 2001 solar proton events. ADV SPACE RES 37, 1678. DOI. ADS.

Kallenrode, M.-B., Wibberenz, G., Kunow, H., Müller-Mellin, R., Stolpovskii, V., Kontor, N.: 1993, Multi-spacecraft observations of particle events and interplanetary shocks during November/December 1982. SOL PHYS 147, 377. DOI. ADS.

Kelly, J., Dalla, S., Laitinen, T.: 2012, Cross-field Transport of Solar Energetic Particles in a Large-scale Fluctuating Magnetic Field. ASTROPHYS J 750, 47. DOI. ADS. 
Klein, K.-L., Trottet, G., Lantos, P., Delaboudinière, J.-P.: 2001, Coronal electron acceleration and relativistic proton production during the 14 July 2000 flare and CME. ASTRON ASTROPHYS 373, 1073. DOI. ADS.

Komori, H.: 1979, An Analysis of Relativistic Cosmic Ray Increase on 22ND November 1977. International Cosmic Ray Conference 4, 19. ADS.

Kóta, J.: 1979, Drift - the Essential Process in Losing Energy. International Cosmic Ray Conference 3, 13. ADS.

Laurenza, M., Consolini, G., Storini, M., Damiani, A.: 2012, A Shannon entropy approach to the temporal evolution of SEP energy spectrum. Astrophysics and Space Sciences Transactions 8, 19. DOI. ADS.

Li, G., Zank, G.P., Verkhoglyadova, O., Mewaldt, R.A., Cohen, C.M.S., Mason, G.M., Desai, M.I.: 2009, Shock Geometry and Spectral Breaks in Large SEP Events. ASTROPHYS J 702, 998. DOI. ADS.

Lin, R.P., Anderson, K.A., Ashford, S., Carlson, C., Curtis, D., Ergun, R., Larson, D., McFadden, J., McCarthy, M., Parks, G.K., Rème, H., Bosqued, J.M., Coutelier, J., Cotin, F., D’Uston, C., Wenzel, K.-P., Sanderson, T.R., Henrion, J., Ronnet, J.C., Paschmann, G.: 1995, A Three-Dimensional Plasma and Energetic Particle Investigation for the Wind Spacecraft. SPACE SCI REV 71, 125. DOI. ADS.

Liu, Y.D., Richardson, J.D., Wang, C., Luhmann, J.G.: 2014, Propagation of the 2012 March Coronal Mass Ejections from the Sun to Heliopause. ASTROPHYS LETT COMM 788, L28. DOI. ADS.

Maia, D.J.F., Gama, R., Mercier, C., Pick, M., Kerdraon, A., Karlický, M.: 2007, The RadioCoronal Mass Ejection Event on 2001 April 15. ASTROPHYS J 660, 874. DOI. ADS.

Marsh, M.S., Dalla, S., Kelly, J., Laitinen, T.: 2013, Drift-induced Perpendicular Transport of Solar Energetic Particles. ASTROPHYS J 774, 4. DOI. ADS.

McCuistion, D., Bengston, C.: 1996, GOES-8 and 9 Mission Launch and Operations Support Overview. In: Guyenne, T.-D. (ed.) Space Mission Operations and Ground Data Systems SpaceOps '96, ESA Special Publication 394, 76. ADS.

Mewaldt, R.A., Looper, M.D., Cohen, C.M.S., Haggerty, D.K., Labrador, A.W., Leske, R.A., Mason, G.M., Mazur, J.E., von Rosenvinge, T.T.: 2012, Energy Spectra, Composition, and Other Properties of Ground-Level Events During Solar Cycle 23. Space Sci Rev 171, 97. DOI. ADS.

Mewaldt, R.A., Cohen, C.M.S., Cook, W.R., Cummings, A.C., Davis, A.J., Geier, S., Kecman, B., Klemic, J., Labrador, A.W., Leske, R.A., Miyasaka, H., Nguyen, V., Ogliore, R.C., Stone, E.C., Radocinski, R.G., Wiedenbeck, M.E., Hawk, J., Shuman, S., von Rosenvinge, T.T., Wortman, K.: 2008, The Low-Energy Telescope (LET) and SEP Central Electronics for the STEREO mission. SPACE SCI REV 136(1-4), 285. DOI. http://link.springer.com/article/ 10.1007/s11214-007-9288-x.

Muraki, Y., Matsubara, Y., Masuda, S., Sakakibara, S., Sako, T., Watanabe, K., Bütikofer, R., Flückiger, E.O., Chilingarian, A., Hovsepyan, G., Kakimoto, F., Terasawa, T., Tsunesada, Y., Tokuno, H., Velarde, A., Evenson, P., Poirier, J., Sakai, T.: 2008, Detection of high-energy solar neutrons and protons by ground level detectors on April 15, 2001. ASTROPART PHYS 29, 229. DOI. ADS.

Onsager, T., Grubb, R., Kunches, J., Matheson, L., Speich, D., Zwickl, R.W., Sauer, H.: 1996, Operational uses of the GOES energetic particle detectors. In: Washwell, E.R. (ed.) GOES8 and Beyond, Society of Photo-Optical Instrumentation Engineers (SPIE) Conference Series 2812, 281. DOI. ADS.

Porsche, H.: 1981, HELIOS mission: Mission objectives, mission verification, selected results. In: Burke, W.R. (ed.) Solar System and its Exploration, ESA Special Publication 164, 43. ADS.

Reames, D.V., Barbier, L.M., Ng, C.K.: 1996, The Spatial Distribution of Particles Accelerated by Coronal Mass Ejection-driven Shocks. ASTROPHYS J 466, 473. DOI. ADS.

Reames, D.V., Kahler, S.W., Ng, C.K.: 1997, Solar Energetic-Particle Spectra and the Structure of Coronal Mass Ejections. International Cosmic Ray Conference 1, 185. ADS.

Richardson, I.G., von Rosenvinge, T.T., Cane, H.V., Christian, E.R., Cohen, C.M.S., Labrador, A.W., Leske, R.A., Mewaldt, R.A., Wiedenbeck, M.E., Stone, E.C.: 2014, ¿25 MeV proton events observed by the High Energy Telescopes on the STEREO A and B spacecraft and/or at Earth during the first seven years of the STEREO mission. SOL PHYS 289(8), 3059. DOI. http://link.springer.com/article/10.1007/s11207-014-0524-8.

Ruffolo, D.: 1995, Effect of adiabatic deceleration on the focused transport of solar cosmic rays. ASTROPHYS J 442, 861. DOI. ADS. 
Rujiwarodom, M., Bieber, J., Droge, W., Everson, P., Pyle, R., Ruffolo, D., Tooprakai, P., Khumlumlert, T.: 2003, Time of Solar Energetic Particle Acceleration on 15-04-2001. In: IAU Joint Discussion, IAU Joint Discussion 7, 35. ADS.

Stone, E.C., Cohen, C.M.S., Cook, W.R., Cummings, A.C., Gauld, B., Kecman, B., Leske, R.A., Mewaldt, R.A., Thayer, M.R., Dougherty, B.L., Grumm, R.L., Milliken, B.D., Radocinski, R.G., Wiedenbeck, M.E., Christian, E.R., Shuman, S., von Rosenvinge, T.T.: 1998, The Solar Isotope Spectrometer for the Advanced Composition Explorer. SPACE SCI REV 86(1-4), 357. DOI. http://link.springer.com/article/10.1023/A:1005027929871.

Torsti, J., Valtonen, E., Lumme, M., Peltonen, P., Eronen, T., Louhola, M., Riihonen, E., Schultz, G., Teittinen, M., Ahola, K., Holmlund, C., Kelh, V., Leppl, K., Ruuska, P., Strmmer, E.: 1995, Energetic particle experiment ERNE. SOL PHYS 162(1-2), 505. DOI. http://link.springer.com/article/10.1007/BF00733438.

Tylka, A.J., Boberg, P.R., Cohen, C.M.S., Dietrich, W.F., Maclennan, C.G., Mason, G.M., Reames, D.V., McGuire, R.E., Ng, C.K.: 2001a, Energy Spectra of Very Large Gradual Solar Particle Events. International Cosmic Ray Conference 8, 3189. ADS.

Tylka, A.J., Cohen, C.M.S., Dietrich, W.F., Maclennan, C.G., McGuire, R.E., Ng, C.K., Reames, D.V.: 2001b, Evidence for Remnant Flare Suprathermals in the Source Population of Solar Energetic Particles in the 2000 Bastille Day Event. ASTROPHYS LETT COMM 558, L59. DOI. ADS.

Tylka, A.J., Boberg, P.R., Cohen, C.M.S., Dietrich, W.F., Maclennan, C.G., Mason, G.M., Ng, C.K., Reames, D.V.: 2002, Flare- and Shock-accelerated Energetic Particles in the Solar Events of 2001 April 14 and 15. ASTROPHYS LETT COMM 581, L119. DOI. ADS.

Vainio, R., Valtonen, E., Heber, B., Malandraki, O.E., Papaioannou, A., Klein, K.-L., Afanasiev, A., Agueda, N., Aurass, H., Battarbee, M., Braune, S., Dröge, W., Ganse, U., Hamadache, C., Heynderickx, D., Huttunen-Heikinmaa, K., Kiener, J., Kilian, P., Kopp, A., Kouloumvakos, A., Maisala, S., Mishev, A., Miteva, R., Nindos, A., Oittinen, T., Raukunen, O., Riihonen, E., Rodríguez-Gasén, R., Saloniemi, O., Sanahuja, B., Scherer, R., Spanier, F., Tatischeff, V., Tziotziou, K., Usoskin, I.G., Vilmer, N.: 2013, The first SEPServer event catalogue $\sim 68-\mathrm{MeV}$ solar proton events observed at $1 \mathrm{AU}$ in 1996-2010. Journal of Space Weather and Space Climate 3(27), A12. DOI. ADS.

von Rosenvinge, T.T., Reames, D.V., Baker, R., Hawk, J., Nolan, J.T., Ryan, L., Shuman, S., Wortman, K.A., Mewaldt, R.A., Cummings, A.C., Cook, W.R., Labrador, A.W., Leske, R.A., Wiedenbeck, M.E.: 2008, The High Energy Telescope for STEREO. SPACE SCI REV 136(1-4), 391. DOI. http://link.springer.com/article/10.1007/s11214-007-9300-5.

Wang, J.-X., Zhou, G.-P., Wen, Y.-Y., Zhang, Y.-Z., Wang, H.-N., Deng, Y.-Y., Zhang, J., Harra, L.K.: 2006, Transequatorial Filament Eruption and Its Link to a Coronal Mass Ejection. CJAA 6, 247. DOI. ADS.

Wang, R., Wang, J.: 2005, Solar Relativistic Proton Fluxes in the Solar Flare of 14 July 2000. In: Dere, K., Wang, J., Yan, Y. (eds.) Coronal and Stellar Mass Ejections, IAU Symposium 226, 381. DOI. ADS

Wiedenbeck, M.E., Mason, G.M., Cohen, C.M.S., Nitta, N.V., Gómez-Herrero, R., Haggerty, D.K.: 2013, Observations of Solar Energetic Particles from ${ }^{3} \mathrm{He}$-rich Events over a Wide Range of Heliographic Longitude. ASTROPHYS J 762, 54. DOI. ADS.

Zhang, M., McKibben, R.B., Lopate, C., Jokipii, J.R., Giacalone, J., Kallenrode, M.-B., Rassoul, H.K.: 2003, Ulysses observations of solar energetic particles from the 14 July 2000 event at high heliographic latitudes. J GEOPHYS RES 108, 1154. DOI. ADS. 